\title{
Hadronic matter under an external magnetic field: In-medium modification of the pion mass
}

\author{
R. M. Aguirre \\ Departamento de Fisica, Facultad de Ciencias Exactas, Universidad Nacional de La Plata, \\ and Instituto de Fisica La Plata, CONICET, La Plata (1900), Argentina
}

(Received 6 October 2016; published 21 April 2017)

\begin{abstract}
The covariant propagator of a fermion with intrinsic magnetic moment interacting with a uniform external magnetic field is presented for finite temperature and baryonic density. The case of a scalar boson is also considered. The final expressions are given in terms of a four-dimensional momentum representation. These results, which take account of the full effect of the magnetic field, are used to evaluate the modification of the pion mass at zero temperature as a function of the density and the magnetic intensity. For this purpose a self-consistent calculation, including one- and two-pion vertices, is employed.
\end{abstract}

DOI: 10.1103/PhysRevD.95.074029

\section{INTRODUCTION}

The dynamics of matter subject to strong magnetic fields has been widely studied in the past [1], and it has received renewed interest due to the analysis of different experimental situations [2].

In recent years, the significative role played by the intrinsic magnetic moments of the hadrons when the thermodynamical behavior of dense nuclear matter under strong magnetic fields is analyzed has been pointed out [3-7].

This conclusion can be made extensive, for instance, to the study of matter created in heavy ion collisions, where very intense magnetic fields have been predicted [8-10]. Experimental evidence of this fact is the preferential emission of charged particles along the direction of the magnetic field for noncentral heavy ion collisions, due to magnetic intensities $e B \sim 10^{2} \mathrm{MeV}^{2}[8]$.

In a different scenario, very dense hadronic matter under strong magnetic fields could be found in certain compact stars, which have generally been included within the magnetar model $[11,12]$. The sustained $\mathrm{x}$-ray luminosity in the soft $(0.5-10 \mathrm{keV})$ or hard $(50-200 \mathrm{keV})$ spectrum, as well as the bursting activity of these objects, are attributed to the dissipation and decay of very strong magnetic fields. The intensity of these fields has been estimated around $10^{15} \mathrm{G}$ at the star surface, but it could reach much higher values in the dense interior of the star. The availability of an increasing amount of precision data opens the question on how well the current theoretical description of hadronic matter can fit this empirical evidence.

A successful description of the dense hadronic environment has been given by a covariant model of the hadronic interaction known as quantum hadrodynamics (QHD) [13]. It has been used to study the structure of neutron stars, and particularly to analyze hadronic matter in the presence of an external magnetic field $[3,4,6,7,14-16]$. The versatility of this formulation allows the inclusion of the intrinsic magnetic moments in a covariant way. Due to the strength of the baryon-meson couplings, the mean field approximation (MFA) is usually employed. Within this approach, the meson fields are replaced by their expectation values and assimilated into a quasiparticle picture of the baryons. Finally, the meson mean values are obtained by solving the classical meson equations, taking as sources the baryonic currents. This scheme is conceptually clear and easy to implement.

The propagators of charged particles in external magnetic fields have been analyzed from different points of view [17-21]. A first attempt to include the full effect of the intrinsic magnetic moments of a Dirac particle propagating in a dense hadronic environment has been presented in Ref. [21]. However, it uses a mixed representation, where position and momentum variables are bound together. The coherence of the approach was tested by evaluating typical currents and densities in nuclear matter.

In the present work, we try to improve that formalism by presenting a representation in terms of only momentum coordinates. Obviously, this fact makes easier the application of diagrammatic procedures. In this sense, our results could be useful to complement recent studies [22-24].

The propagator of Dirac particles subject to a uniform external magnetic field, which includes the full effect of its magnetic moments, is used to evaluate corrections to the pion propagator in a dense nuclear environment. In particular, we evaluate the meson polarization in conditions appropriate to defining the effective pion mass. We analyze a wide range of densities $0<n_{B}<3 n_{0}$, with $n_{0}$ being the saturation density of nuclear matter, and we concentrate on very strong magnetic fields, $B \geq 10^{17} \mathrm{G}$. Two different isospin compositions of nuclear matter are considered, pure neutron matter and symmetric nuclear matter.

The behavior of the pion polarization, and particularly of its effective mass, has recently been studied for low matter density and a wide range of magnetic intensities [25,26]. However, the intrinsic nucleon magnetic moments are not considered in these works. In contrast, in our analysis, the 
variation of the neutral pion mass in pure neutron matter is an effect exclusively due to the neutron magnetic moment.

This work is organized as follows: In the next section, a summary of the findings of Ref. [21] is presented, and further development is made to derive a four-momentum representation for the propagator. Since we are interested in evaluating the effects on the pion propagation, we give a brief overview of the Green function for a charged spin-zero meson in Sec. III. The evaluation of the in-medium pion polarization and the definition of its effective mass are given in Sec. IV. We devote Sec. V to the discussion of the results. Finally, the conclusions are shown in Sec. VI. Certain details of the mathematical elaboration are transferred to the appendixes.

\section{IN-MEDIUM PROPAGATOR OF A DIRAC FIELD WITH INTRINSIC MAGNETIC MOMENT}

A preliminary version of the results of this section was presented in Ref. [21]. For the sake of completeness, we give here an overview of the procedure.

The interaction of a spin-1/2 fermion with a uniform magnetic field is described by the Lagrangian density

$$
\mathcal{L}=\bar{\Psi}\left[\gamma_{\mu}\left(i \partial^{\mu}-q A^{\mu}\right)-m+\frac{\kappa}{2} \sigma^{\mu \nu} \mathcal{F}_{\mu \nu}\right] \Psi,
$$

where $\mathcal{F}^{\mu \nu}=\partial^{\mu} A^{\nu}-\partial^{\nu} A^{\mu}$ and $\sigma^{\mu \nu}=i\left[\gamma^{\mu}, \gamma^{\nu}\right] / 2$. For simplicity, the case of a uniform external magnetic field of magnitude $B$ along the $z$-axis is considered, for which $A^{\mu}=g_{2}^{\mu} B x$.

For charged particles of positive energies $E_{n s}$, an exact solution in mixed position and momentum coordinates can be written as

$$
\phi_{n s p_{y} p_{z}}^{(+)}(\xi, y, z)=e^{i\left(p_{y} y+p_{z} z\right)} e^{-\xi^{2} / 2} u_{n s p_{z}}(\xi),
$$

where the index $s=1,-1$ stands for the spin projection along the magnetic field direction, and $n \geq 1$ denotes the quantized Landau levels. We have also used

$$
u_{n s p_{z}}(\xi)=N_{n s}\left(\begin{array}{c}
H_{n}(\xi) \\
\frac{2 n s p_{z} \sqrt{q B} i}{\left(\Delta_{n}+s m\right)\left(E_{n s}+s \Delta_{n}-\kappa B\right)} H_{n-1}(\xi) \\
\frac{p_{z}}{E_{n s}+s \Delta_{n}-\kappa B} H_{n}(\xi) \\
-\frac{2 n s \sqrt{q B} i}{\Delta_{n}+s m} H_{n-1}(\xi)
\end{array}\right)
$$

and

$$
\begin{gathered}
\xi=\left(-p_{y}+q B x\right) / \sqrt{q B}, \\
\Delta_{n}=\sqrt{m^{2}+2 n q B}, \\
E_{n s}=\sqrt{p_{z}^{2}+\left(\Delta_{n}-s \kappa B\right)^{2}}, \\
N_{n s}^{2}=\frac{\sqrt{q B / \pi}}{(2 \pi)^{2} 2^{n+2} n !} \frac{\left(\Delta_{n}+s m\right)\left(E_{n s}+s \Delta_{n}-\kappa B\right)}{m\left(\Delta_{n}-s \kappa B\right)} ;
\end{gathered}
$$

$H_{n}$ stands for the Hermite polynomials.
The minimum energy eigenstate corresponds to $\phi_{0 p_{y} p_{z}}^{(+)}(\xi, y, z)=e^{i\left(p_{y} y+p_{z} z\right)} e^{-\xi^{2} / 2} u_{0 p_{z}}$, with

$$
u_{0 p_{z}}=N_{0}\left(\begin{array}{c}
1 \\
0 \\
\frac{p_{z}}{E_{0}+m-\kappa B} \\
0
\end{array}\right)
$$

and

$$
\begin{gathered}
E_{0}=\sqrt{p_{z}^{2}+(m-\kappa B)^{2}}, \\
N_{0}^{2}=\frac{\sqrt{q B / \pi}}{2(2 \pi)^{2}} \frac{\left(E_{0}+m-\kappa B\right)}{(m-\kappa B)} .
\end{gathered}
$$

The antiparticle states correspond to the eigenvalues $-E_{n s}$ and have eigenfunctions

$$
\phi_{n s p_{y} p_{z}}^{(-)}(\xi, y, z)=e^{-i\left(p_{y} y+p_{z} z\right)} e^{-\eta^{2} / 2} v_{n s p_{z}}(\eta),
$$

with

$$
v_{n s p_{z}}(\eta)=N_{n s}\left(\begin{array}{c}
\frac{p_{z}}{E_{n s}+s \Delta_{n}-\kappa B} H_{n}(\eta) \\
\frac{2 n s \sqrt{q B} i}{\Delta_{n}+s m} H_{n-1}(\eta) \\
H_{n}(\eta) \\
\frac{-2 n s p_{z} \sqrt{q B} i}{\left(\Delta_{n}+s m\right)\left(E_{n s}+s \Delta_{n}-\kappa B\right)} H_{n-1}(\eta)
\end{array}\right)
$$

where $\eta=\left(p_{y}+q B x\right) / \sqrt{q B}$ and $n \geq 1$. The special case $n=0$ has energy $-E_{0}$ and wave function $\phi_{0 p_{y} p_{z}}^{(-)}(\eta, y, z)=$ $e^{-i\left(p_{y} y+p_{z} z\right)} e^{-\eta^{2} / 2} v_{0 p_{z}}$, with

$$
v_{0 p_{z}}=N_{0}\left(\begin{array}{c}
\frac{p_{z}}{E_{0}+m-\kappa B} \\
0 \\
1 \\
0
\end{array}\right) \text {. }
$$

For neutral particles $(q=0)$, the results are simpler. The particle states are described by

$$
\phi_{\vec{p} s}^{(+)}(\vec{r})=e^{i \vec{p} \cdot \vec{r}} u_{\vec{p} s}
$$

with

$$
u_{\vec{p} s}=N_{\vec{p} s}\left(\begin{array}{c}
1 \\
\frac{-s\left(p_{x}+i p_{y}\right) p_{z}}{(\Delta+s m)\left(E_{\vec{p} s}+s \Delta-\kappa B\right)} \\
\frac{p_{z}}{E_{\vec{p} s}+s \Delta-\kappa B} \\
\frac{s\left(p_{x}+i p_{y}\right)}{\Delta+s m}
\end{array}\right)
$$

and

$$
E_{\vec{p} s}=\sqrt{p_{z}^{2}+(\Delta-s \kappa B)^{2}}
$$




$$
\begin{gathered}
\Delta=\sqrt{m^{2}+p_{x}^{2}+p_{y}^{2}} \\
N_{\vec{p} s}^{2}=\frac{1}{4(2 \pi)^{3}} \frac{(\Delta+s m)\left(E_{\vec{p} s}+s \Delta-\kappa B\right)}{m(\Delta-s \kappa B)} .
\end{gathered}
$$

On the other hand, the antiparticle states have energies $-E_{\vec{p} s}$, and eigenfunctions $\phi_{\vec{p} s}^{(-)}(\vec{r})=e^{-i \vec{p} \cdot \vec{r}} v_{\vec{p} s}$, with

$$
v_{\vec{p} s}=N_{\vec{p} s}\left(\begin{array}{c}
\frac{p_{z}}{E_{\vec{p} s}+s \Delta-\kappa B} \\
\frac{s\left(p_{x}+i p_{y}\right)}{\Delta+s m} \\
1 \\
\frac{-s\left(p_{x}+i p_{y}\right) p_{z}}{(\Delta+s m)\left(E_{\vec{p} s}+s \Delta-\kappa B\right)}
\end{array}\right) .
$$

In the next step, we make a canonical expansion of the fermion quantum fields using the eigenfunctions just described. These fields are used to evaluate the in-medium causal propagator [18]

$$
i G_{\alpha \beta}\left(x^{\prime}, x\right)=\left\langle T \Psi_{\alpha}\left(x^{\prime}\right) \bar{\Psi}_{\beta}(x)\right\rangle
$$

Here the angular brackets must be regarded as a statistical mean value, as obtained, for instance, by evaluating the trace with the density matrix of the system.

Using such a procedure, a mixed-coordinates representation has been obtained for the covariant propagator of a charged Dirac particle [21]:

$$
\begin{aligned}
G_{\alpha \beta}\left(t^{\prime}, \vec{r}, t, \vec{r}\right)= & \sqrt{\frac{q B}{\pi}} \int \frac{d p_{0} d p_{y} d p_{z}}{(2 \pi)^{3}} e^{-i p_{0}\left(t^{\prime}-t\right)} e^{i\left[p_{y}\left(y^{\prime}-y\right)+p_{z}\left(z^{\prime}-z\right)\right]} e^{-\left(\xi^{\prime 2}+\xi^{2}\right) / 2} \\
& \times\left\{\Lambda_{\alpha \beta}^{0}\left[\frac{1}{p_{0}^{2}-E_{0}^{2}+i \epsilon}+2 \pi i n_{F}\left(p_{0}\right) \delta\left(p_{0}^{2}-E_{0}^{2}\right)\right]+\sum_{n, s} \frac{\Delta_{n}+s m}{2^{n+1} n ! \Delta_{n}} \Lambda_{\alpha \beta}^{n s}\left(\xi^{\prime}, \xi\right)\right. \\
& \left.\times\left[\frac{1}{p_{0}^{2}-E_{n s}^{2}+i \epsilon}+2 \pi i n_{F}\left(p_{0}\right) \delta\left(p_{0}^{2}-E_{n s}^{2}\right)\right]\right\},
\end{aligned}
$$

where

$$
\begin{aligned}
\Lambda^{0} & =(\not{u}+m-\kappa B) \Pi^{(+)} \\
\Lambda^{n s} & =\left[\left(\not{u}+s \Delta_{n}-\kappa B\right) H_{n}\left(\xi^{\prime}\right)+i \frac{m-s \Delta_{n}}{\sqrt{q B}}\left(\not{u}-s \Delta_{n}+\kappa B\right) \gamma^{1} H_{n-1}\left(\xi^{\prime}\right)\right]\left[\Pi^{(+)} H_{n}(\xi)+i \frac{m-s \Delta_{n}}{\sqrt{q B}} \gamma^{1} \Pi^{(-)} H_{n-1}(\xi)\right],
\end{aligned}
$$

and $\ddot{u}=p_{0} \gamma^{0}-p_{z} \gamma^{3}, \Pi^{( \pm)}=\left(1 \pm i \gamma^{1} \gamma^{2}\right) / 2, \xi^{\prime}=\left(-p_{y}+q B x^{\prime}\right) / \sqrt{q B}$.

For the neutral fermions, it is

$$
G_{\alpha \beta}\left(x^{\prime}, x\right)=\sum_{s} \int \frac{d p^{4}}{(2 \pi)^{4}} e^{-i p^{\mu}\left(x_{\mu}-x_{\mu}\right)} \Lambda_{\alpha \beta}^{s}\left[\frac{1}{p_{0}^{2}-E_{\vec{p} s}^{2}+i \epsilon}+2 \pi i n_{F}\left(p_{0}\right) \delta\left(p_{0}^{2}-E_{\vec{p} s}^{2}\right)\right],
$$

where

$$
\Lambda^{s}=\frac{s}{2 \Delta} i \gamma^{1} \gamma^{2}\left[u+i \gamma^{1} \gamma^{2}(s \Delta-\kappa B)\right]\left(\not b+m+i s \Delta \gamma^{1} \gamma^{2}\right)
$$

and the notation $x=-p_{x} \gamma^{1}-p_{y} \gamma^{2}$ is introduced.

The formal difference between the results for the neutral and charged fermions must be noted. In the first case, a pure momentum representation can be easily extracted from Eq. (18). This is not the case for Eq. (17), from which a mixed-coordinate $\left(p_{0}, p_{y}, p_{z} ; x, x^{\prime}\right)$ representation can be deduced at most. This fact is related to the particular gauge chosen for the electromagnetic field. Obviously, this is an undesirable flaw for some specific applications-for instance, diagrammatic expansions.

The problem of the gauge invariance of fermion propagators has been discussed a long time ago [17]. By following such studies, a transformation is applied to Eq. (17) which leads to the following decomposition:

$$
G\left(x^{\prime}, x\right)=e^{i \Phi} \int \frac{d^{4} p}{(2 \pi)^{4}} e^{-i p^{\mu}\left(x_{\mu}^{\prime}-x_{\mu}\right)}\left[G_{0}(p)+\sum_{n, s} G_{n, s}(p)\right],
$$

with

$G_{0}(p)=2 e^{-p_{\perp}^{2} / q B} \Lambda^{0}\left[\frac{1}{p_{0}^{2}-E_{0}^{2}+i \epsilon}+2 \pi i n_{F}\left(p_{0}\right) \delta\left(p_{0}^{2}-E_{0}^{2}\right)\right]$, 


$$
\begin{aligned}
G_{n s}(p)= & (-1)^{n} e^{-p_{\perp}^{2} / q B} \frac{\Delta_{n}+s m}{\Delta_{n}}\left\{\left(\not{u}-\kappa B+s \Delta_{n}\right) \Pi^{(+)} L_{n}\left(2 p_{\perp}^{2} / q B\right)-\left(\not{u}+\kappa B-s \Delta_{n}\right) \Pi^{(-)} \frac{s \Delta_{n}-m}{s \Delta_{n}+m} L_{n-1}\left(2 p_{\perp}^{2} / q B\right)\right. \\
& \left.+\left[\ddot{u}+i \gamma_{1} \gamma_{2}\left(s \Delta_{n}-\kappa B\right)\right] i \gamma^{1} \gamma^{2} \not \frac{s \Delta_{n}-m}{2 p_{\perp}^{2}}\left[L_{n}\left(2 p_{\perp}^{2} / q B\right)-L_{n-1}\left(2 p_{\perp}^{2} / q B\right)\right]\right\} \\
& \times\left[\frac{1}{p_{0}^{2}-E_{n s}^{2}+i \epsilon}+2 \pi i n_{F}\left(p_{0}\right) \delta\left(p_{0}^{2}-E_{n s}^{2}\right)\right] ;
\end{aligned}
$$

here $L_{m}$ stands for the Laguerre polynomial of order $m$, and $p_{\perp}^{2}=p_{x}^{2}+p_{y}^{2}$ is used. The phase factor $\Phi=$ $q B\left(x+x^{\prime}\right)\left(y^{\prime}-y\right) / 2$ embodies the gauge fixing. For mathematical details, see Appendix A.

\section{IN-MEDIUM PROPAGATOR OF A CHARGED SCALAR BOSE FIELD}

The covariant propagator of a charged scalar field in the presence of an external magnetic field has been studied in the past, including the method of eigenfunctions expansion [19]. We present here a procedure which renders the propagator into a four-dimensional momentum representation.

The meson field $\phi(x)$ interacting with a electromagnetic field $A_{\mu}(x)$ is described by the Lagrangian

$$
\mathcal{L}=\left(\partial^{\mu}-i e A^{\mu}\right) \varphi^{\dagger}\left(\partial_{\mu}+i e A_{\mu}\right) \varphi-m^{2} \varphi^{\dagger} \varphi .
$$

We choose the gauge as in Sec. II. The eigenfunctions are

$$
\varphi_{n}(x)=\mathcal{N} e^{-i\left(\omega_{n} t-p_{y} y-p_{z} z\right)} e^{-\xi^{2} / 2} H_{n}(\xi),
$$

with $\omega_{n}=\sqrt{m^{2}+p_{z}^{2}+2(n+1) q B}, \xi=\sqrt{q B}\left(x-p_{y} / q B\right)$, and $\mathcal{N}^{2}=\sqrt{q B / \pi} / 2^{n} n$ ! An expansion of the quantum field is proposed as

$$
\phi(x)=\sum_{n, l} \int \frac{d p_{z}}{2 \pi \omega_{n}}\left[\varphi_{n l} a_{n l}\left(p_{z}\right)+\varphi_{n l}^{*} b_{n l}^{\dagger}\left(p_{z}\right)\right],
$$

with canonical commutation relations for the creation and destruction operators. Using the standard definition of the propagator $i \Delta\left(x, x^{\prime}\right)=\left\langle T \phi(x) \phi^{\dagger}\left(x^{\prime}\right)\right\rangle$, where angular brackets stand for a statistical expectation value, we obtain

$\left.\left.\Delta\left(x, x^{\prime}\right)=\sum_{n} \mathcal{N}^{2} \int \frac{d p_{0} d p_{y} d p_{z}}{(2 \pi)^{3}} e^{-i p_{0}\left(t-t^{\prime}\right)+i p_{y}\left(y-y^{\prime}\right)+i p_{z}\left(z-z^{\prime}\right)} H_{n}^{(} \xi\right) H_{n}^{(} \xi^{\prime}\right) e^{-\left(\xi^{2}+\xi^{\prime 2}\right) / 2}\left[\frac{1}{p_{0}^{2}-\omega_{n}^{2}+i \varepsilon}+2 \pi i \delta\left(p_{0}^{2}-\omega_{n}^{2}\right) n_{B}\left(p_{0}\right)\right]$,

where $n_{B}$ is the Bose distribution function, and we use the identity

$$
\frac{1}{2 \omega_{n}}\left[\Theta\left(t^{\prime}-t\right) e^{i \omega_{n}\left(t-t^{\prime}\right)}+\Theta\left(t-t^{\prime}\right) e^{-i \omega_{n}\left(t-t^{\prime}\right)}\right]=\frac{i}{2 \pi} \int \frac{d p_{0}}{2 \pi} \frac{e^{-i p_{0}\left(t-t^{\prime}\right)}}{p_{0}^{2}-\omega_{n}^{2}+i \varepsilon}
$$

in order to unify particle and antiparticle notation.

In the first place, we perform the integration over $p_{y}$,

$$
\int d p_{y} \mathcal{N}^{2} e^{i p_{y}\left(y-y^{\prime}\right)} e^{-\left(\xi^{2}+\xi^{2}\right) / 2} H_{n}^{(\xi)} H_{n}^{\left(\xi^{\prime}\right)}=q B e^{i \Phi} e^{-q B R^{2} / 4} L_{n}\left(q B R^{2} / 2\right),
$$

with the help of Eq. 7.377 of Ref. [27], where $R=\sqrt{\left(x-x^{\prime}\right)^{2}+\left(y-y^{\prime}\right)^{2}}$ is used. The right-hand side can be rewritten in terms of a bidimensional momentum integral by using Eq. (A5). Hence, we finally obtain

$$
\begin{aligned}
\Delta\left(x, x^{\prime}\right) & =e^{i \Phi} \int \frac{d^{4} p}{(2 \pi)^{4}} e^{-i p^{\mu}\left(x-x^{\prime}\right)_{\mu}} \Delta(p), \\
\Delta(p) & =2 \sum_{n}(-1)^{n} e^{-p_{\perp}^{2} / q B} L_{n}\left(2 p_{\perp}^{2} / q B\right)\left[\frac{1}{p_{0}^{2}-\omega_{n}^{2}+i \varepsilon}+2 \pi i \delta\left(p_{0}^{2}-\omega_{n}^{2}\right) n_{B}\left(p_{0}\right)\right] .
\end{aligned}
$$

The quantities $\Phi$ and $p_{\perp}$ were defined at the end of Sec. II.

\section{PION EFFECTIVE MASS IN THE NUCLEAR MEDIUM UNDER A UNIFORM MAGNETIC FIELD}

In this section, we consider the hadronic interaction in the presence of a uniform external magnetic field. It is described by a QHD model, where baryons interact with pions and neutral mesons $\sigma$ and $\omega$. The Langragian density is 


$$
\begin{aligned}
\mathcal{L}= & \sum_{a=n, p} \bar{\Psi}^{a}\left[\gamma _ { \mu } \left(i \partial^{\mu}-q_{a} A^{\mu}+g_{w} \omega^{\mu}-\frac{g_{A}}{2 f_{\pi}} \gamma_{5} \boldsymbol{\tau} \cdot \partial^{\mu} \boldsymbol{\phi}\right.\right. \\
& \left.\left.-\frac{1}{4 f_{\pi}^{2}} \boldsymbol{\tau} \cdot \boldsymbol{\phi} \times \partial^{\mu} \boldsymbol{\phi}\right)-m_{0}+g_{s} \sigma+\frac{\kappa}{2} \sigma^{\mu \nu} \mathcal{F}_{\mu \nu}\right] \Psi^{a} \\
& -\frac{1}{4} \mathcal{F}_{\mu \nu} \mathcal{F}^{\mu \nu}+\frac{1}{2}\left(\partial_{\mu} \sigma \partial^{\mu} \sigma-m_{\sigma}^{2} \sigma^{2}\right)-\frac{1}{4} \Omega_{\mu \nu} \Omega^{\mu \nu} \\
& +\frac{1}{2} m_{\omega}^{2} \omega_{\mu} \omega^{\mu}+\frac{1}{2} \partial_{\mu} \phi^{0} \partial^{\mu} \phi^{0} \\
& +\left(\partial^{\mu}-i e A^{\mu}\right) \phi^{-}\left(\partial_{\mu}+i e A_{\mu}\right) \phi^{+}-\frac{1}{2} m_{\pi}^{2} \boldsymbol{\phi} \cdot \boldsymbol{\phi},
\end{aligned}
$$

where $\Omega^{\mu \nu}=\partial^{\mu} \omega^{\nu}-\partial^{\nu} \omega^{\mu}$, and only protons and neutrons have been included. In this approach, the fundamental state of matter is given by a MFA, which is equivalent to including the tadpole diagram [see Fig. 1(a)] in a selfconsistent solution but neglecting divergent contributions coming from the Dirac sea. At this step, it is assumed that meson propagation is not modified by the hadronic interaction. The effect of the magnetic field, instead, is fully included for both meson and nucleon propagators. This means that fermionic lines in the diagram of Fig. 1(a) correspond to either Eqs. (18)-(19) or Eqs. (21)-(22).

It can be verified that pions do not contribute to the tadpole diagram, since the pion-nucleon vertices depend on the transferred pion momentum. Furthermore, the neutral

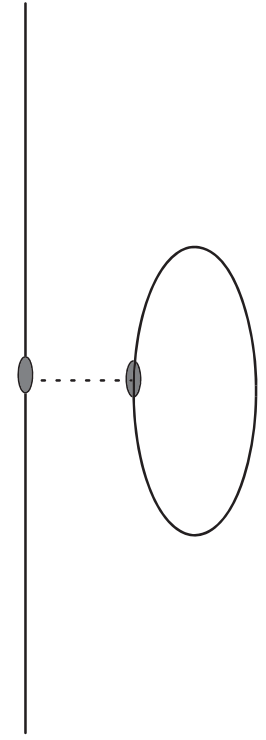

(a)

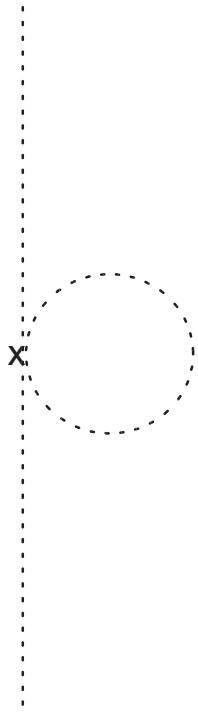

(b)

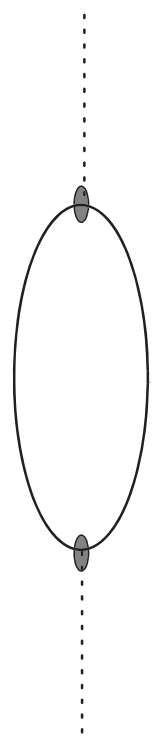

(c)
FIG. 1. The diagrams included in our Dyson-Schwinger calculations. Solid lines represent fermion propagators, while dashed lines represent meson propagators. (a) The tadpole diagram contributing to the mean field approach to the nucleon self-energy. (b) The Weinberg-Tomozawa contribution to the pion polarization. (c) The one-pion exchange diagram for the pion polarization. mesons $\sigma, \omega$, and $\pi^{0}$ are not affected directly by the magnetic field.

It is well known that in QHD models, the MFA leads to a quasiparticle picture for nucleons, where the mass and energy spectra are modified, according to $m=m_{0}-g_{s} \sigma_{0}$, $p_{0}=g_{w} w_{0} \pm E$, with $E$ being one of the eigenvalues shown in Eqs. (5), (8), or (13). The quantities $\sigma_{0}, w_{0}$ correspond to the in-medium expectation values of the $\sigma$ and timelike component of $\omega$ mesons [13], $w_{0}=g_{\omega} n_{B} / m_{\omega}^{2}$ and $\sigma_{0}=g_{\sigma} n_{s} / m_{\sigma}^{2}$. The baryonic number $\left(n_{B}\right)$ and scalar $\left(n_{s}\right)$ densities can be decomposed into their neutron and proton components:

$$
\begin{aligned}
n_{B}^{(n)}= & \sum_{s} \int \frac{d p^{3}}{(2 \pi)^{3}}\left[n_{F}\left(E_{\vec{p} s}\right)-n_{F}\left(-E_{\vec{p} s}\right)\right], \\
n_{B}^{(p)}= & \frac{q B}{2 \pi^{2}} \int d p_{z}\left\{\left[n_{F}\left(E_{0}\right)-n_{F}\left(-E_{0}\right)\right]\right. \\
& \left.+\sum_{n, s}\left[n_{F}\left(E_{n s}\right)-n_{F}\left(-E_{n s}\right)\right]\right\}, \\
n_{s}^{(n)}= & \sum_{s} \int \frac{d p^{3}}{(2 \pi)^{3}} \frac{\Delta+s \kappa_{n} B}{E_{\vec{p} s} \Delta}\left[n_{F}\left(E_{\vec{p} s}\right)+n_{F}\left(-E_{\vec{p} s}\right)\right], \\
n_{s}^{(n)}= & \frac{q B}{2 \pi^{2}} \int d p_{z}\left\{\frac{m+\kappa_{p} B}{E_{0}}\left[n_{F}\left(E_{0}\right)+n_{F}\left(-E_{0}\right)\right]\right. \\
& \left.+m \sum_{n, s} \frac{\Delta_{n}+s \kappa_{p} B}{E_{n s} \Delta_{n}}\left[n_{F}\left(E_{n s}\right)+n_{F}\left(-E_{n s}\right)\right]\right\} .
\end{aligned}
$$

Therefore, at the end of the calculations, we formally recover similar expressions for the nucleon propagators as given in Sec. II, but with the following modifications: (i) the nucleon vacuum mass is replaced by the in-medium effective mass, and (ii) the variable $p_{0}$ must be replaced by $\tilde{p}_{0}=p_{0}-g_{w} w$. For practical applications, the last point is equivalent to replacing the thermodynamical chemical potential $\mu$ with the effective potential $\tilde{\mu}=\mu-g_{w} w$.

In the next step, we study the effects of the hadronic interaction on the meson properties. In particular, we consider the modification of the pion mass. For this purpose, we evaluate the pion polarization insertion due to Figs. 1(b) and 1 (c) corresponding to the one-loop approximation. The diagram in Fig. 1(b) comes from the Weinberg-Tomozawa term and corresponds to a first-order correction.

It gives nonzero contributions only for the nondiagonal channel $(1,2)$ of the Hermitian pion fields

$\Pi_{a b}^{\mathrm{WT}}(p)=-\frac{\varepsilon_{3 a b}}{2 f_{\pi}^{2}} p_{\mu} \sum_{c} \tau_{3}^{c c} \int \frac{d^{4} q}{(2 \pi)^{4}} \operatorname{Tr}\left\{\gamma^{\mu} G^{(c)}(q)\right\}$,

where the sum runs over protons $(c=1)$ and neutrons $(c=2)$. Using the nucleon propagators constructed in the MFA and specializing for the conditions of interest for our calculations, we finally obtain 


$$
\Pi_{ \pm}^{\mathrm{WT}}\left(p_{0}, \boldsymbol{p}=0\right)= \pm \frac{p_{0}}{2 f_{\pi}^{2}}\left(n_{B}^{(n)}-n_{B}^{(p)}\right),
$$

whereas $\Pi_{0}^{\mathrm{WT}}(p)=0$.

The diagram in Fig. 1(c) corresponds to the pseudovector one-pion vertex (OPV); it is a second order correction,

$$
\begin{aligned}
i \Pi^{\mathrm{OPV}}(p)= & \left(\frac{g_{A}}{2 f_{\pi}}\right)^{2} p_{\mu} p_{\nu} \int \frac{d^{4} q}{(2 \pi)^{4}} \\
& \times \operatorname{Tr}\left\{\gamma^{\mu} \gamma_{5} G^{(a)}(q) \gamma^{\nu} \gamma_{5} G^{(b)}(q-p)\right\} .
\end{aligned}
$$

It is understood that for the neutral pion, a sum over $a=b$ must be done; for the positively charged pion, the sum is $a=p, b=n$; and finally, $a=n, b=p$ corresponds to the negatively charged pion. Explicit expressions for the polarizations $\Pi_{0}^{\mathrm{OPV}}, \Pi_{ \pm}^{\mathrm{OPV}}$ evaluated at $\mathbf{p}=0$ are shown in Appendix B.

In a Dyson-Schwinger approach, the poles of the pion propagator are modified by the polarization insertion. For charged pions and for each Landau level, they are given by $p_{0}^{2}-\omega_{n}^{2}-\Pi_{ \pm}(p)=0$. For neutral pions instead, they are defined by $p_{0}^{2}-\left(m_{\pi}^{2}+\boldsymbol{p}^{2}\right)-\Pi_{0}(p)=0$. Hence, the inmedium effective mass is defined as the solutions of the following equation for $p_{0}$ :

$$
p_{0}^{2}-m_{\pi}^{2}-\Pi\left(p_{0}, \boldsymbol{p}=0\right)=0 .
$$

It must be noted that the term $2 n q B$ coming from the quantized Landau states for the charged pions has not been included in this definition.

As was already mentioned, for charged pions the polarization insertion in Eq. (27) is a sum $\Pi_{ \pm}^{\mathrm{WT}}+\Pi_{ \pm}^{\mathrm{OPV}}$, whereas for the neutral pion there is only one contribution, $\Pi_{0}^{\mathrm{OPV}}$.

\section{RESULTS AND DISCUSSION}

In this section, we solve Eq. (27) for different situations of physical interest. We consider here very strong magnetic fields $10^{16}-10^{19} \mathrm{G}$, and matter at zero temperature and baryonic densities below $0.45 \mathrm{fm}^{-3}$. We also take the isospin composition of matter as a variable and examine two different situations: (i) symmetric nuclear matter $n_{B}^{(p)}=n_{B}^{(n)}$, and (ii) pure neutron matter $n_{B}^{(p)}=0$. In the first case, there is no contribution from $\Pi_{ \pm}^{\mathrm{WT}}$, as can be seen from Eq. (25).

In first place, we take a look of the thermodynamical state of matter at zero temperature, within the MFA. The results obtained are used to evaluate the medium-dependent parameters of the fermionic propagators. As a second step, the polarization insertion for pions is constructed and its effective mass is examined.

\section{A. Thermodynamics of the magnetized hadronic medium}

For a given magnetic intensity, baryonic density, and isospin composition, the equilibrium state of matter corresponds to a minimum of the energy of the system. In this state, each isospin component acquires a global spin polarization, induced by the external magnetic field. Furthermore, the system exhibits a weak magnetization. In this section, we examine the thermodynamical properties of the equilibrium state.

In Fig. 2, we present the energy per particle (with subtraction of the nucleon mass $m_{0}$ ) as a function of the density for several magnetic intensities. In symmetric matter, a minimum or saturation point is found, whose energy decreases with the intensity of the magnetic field.

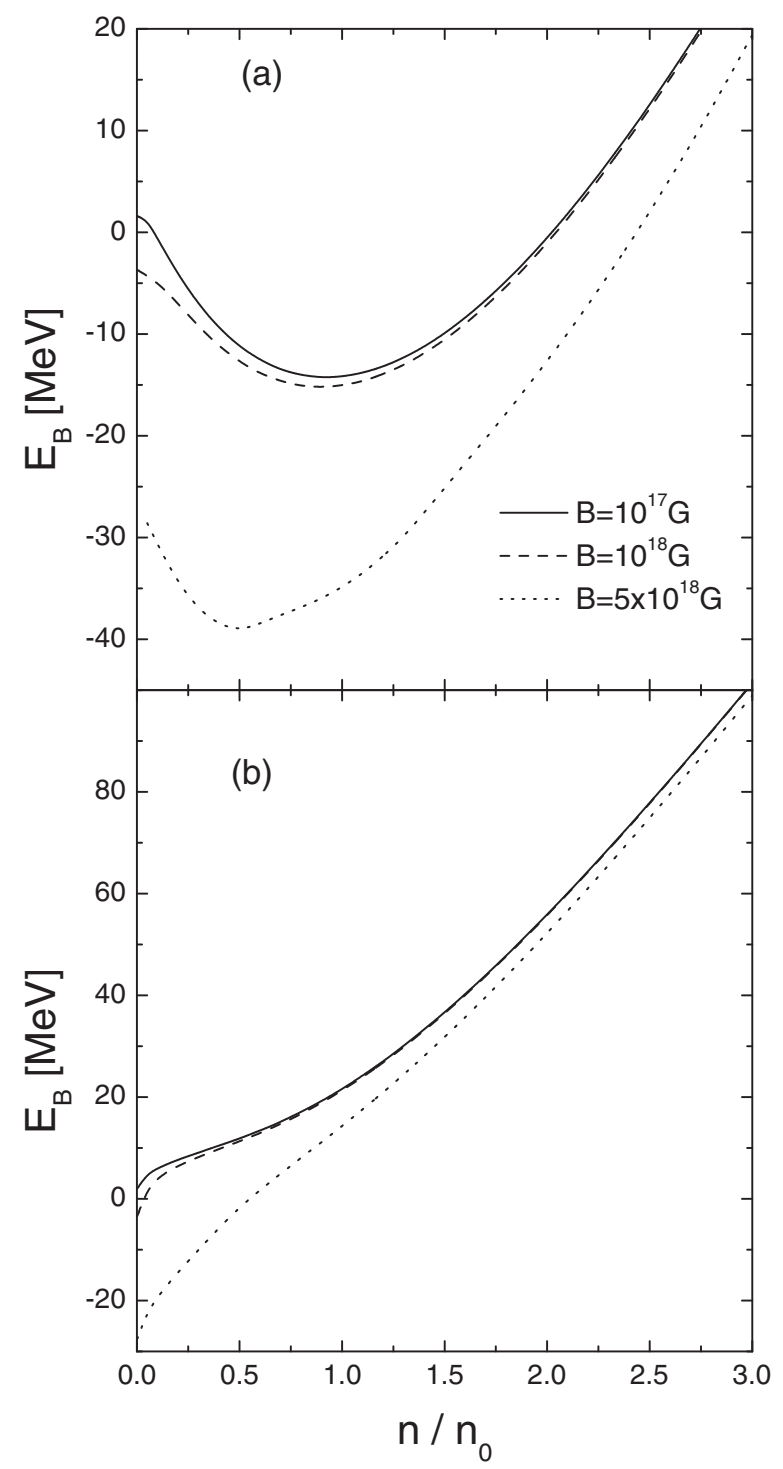

FIG. 2. The energy per particle (with the rest mass subtracted) for (a) symmetric nuclear matter and (b) pure neutron matter, as a function of the density for different magnetic intensities. 
The fact that nuclear matter is more strongly bound as the magnitude of the external field grows has been remarked on in different studies [3,5-7]. For pure neutron matter, instead, a monotonous increase is found. It deserves to be mentioned that at low densities, neutron matter becomes bound, a feature emphasized as $B$ increases [4,5].

Next, in Fig. 3, we analyze the spin polarization of each isospin component as a function of the density. The quantity $W^{(a)}=\left(n_{\text {up }}^{(a)}-n_{\text {down }}^{(a)}\right) / n_{B}^{(a)}$, with $a=p, n$, gives a statistical measure of the fraction of particles with spin polarized in the direction of the field or in opposition to it. For $B>10^{16} \mathrm{G}$, both isospin components are completely polarized at very low densities. There is an abrupt change of polarization for the weaker intensities, while the plateau of

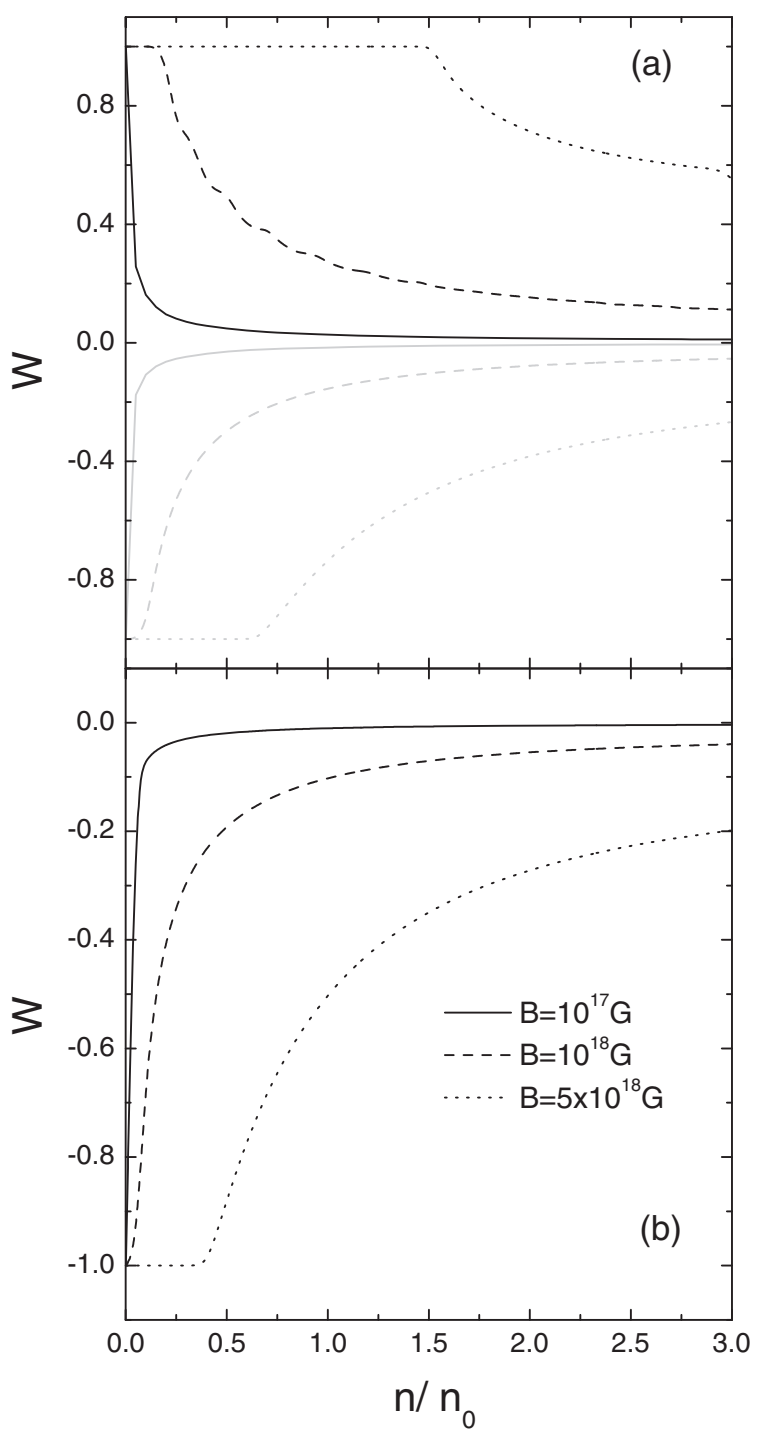

FIG. 3. The degree of polarization in (a) symmetric nuclear matter and (b) pure neutron matter, as a function of the density for different magnetic intensities. In the upper panel, black lines correspond to the proton component, and gray lines correspond to the neutron case. complete polarization is extended in density as the external field grows. In this sense, the response of the proton component seems to be more intense than the neutron one. As a special situation, it can be seen that the curve corresponding to $W^{(p)}$ for symmetric matter at $B=10^{18} \mathrm{G}$ shows several irregularities due to the thresholds in the occupation of different Landau levels. The case $B=$ $10^{16} \mathrm{G}$ has not been included in Figs. 2 and 3, since it is indistinguishable from the $B=10^{17} \mathrm{G}$ curve for the scale shown. In both figures, there is an apparent difference in the qualitative behavior of the $B=5 \times 10^{18} \mathrm{G}$ curves, which can be attributed to the intrinsic magnetic moments [3,6,7], insofar as $\kappa_{a} B \sim 1$.

The effective chemical potential for neutrons and protons as a function of density is exhibited in Fig. 4. It can be appreciated that $\tilde{\mu}$ is a decreasing function, even though the thermodynamical potential $\mu$ increases monotonously, as it corresponds to a thermodynamical equilibrium state. This is a consequence of the faster growth of the mean field value $\omega_{0}$. Only the case $B=5 \times 10^{18} \mathrm{G}$ is shown in this figure, but a similar trend is obtained for other intensities.

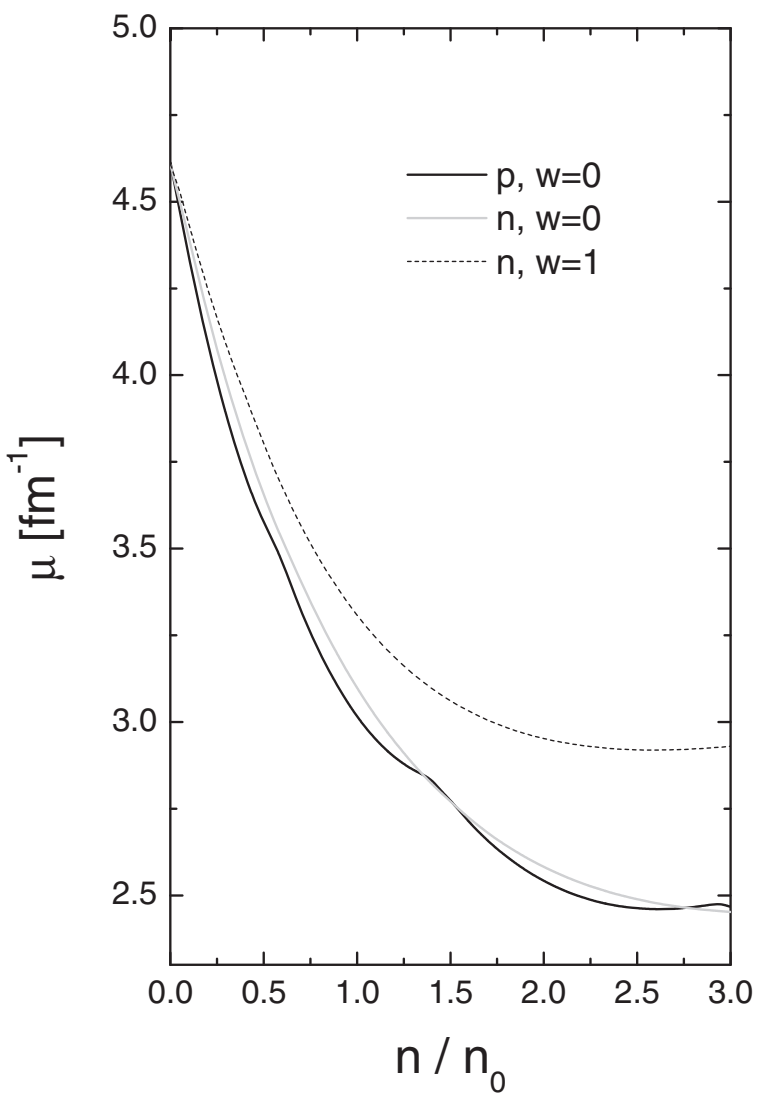

FIG. 4. The effective chemical potential as a function of the density for $B=5 \times 10^{18} \mathrm{G}$. Here $w=\left(n_{B}^{(n)}-n_{B}^{(p)}\right) /\left(n_{B}^{(n)}+\right.$ $\left.n_{B}^{(p)}\right)$ is used. Solid lines correspond to symmetric nuclear matter, and dashed lines correspond to pure neutron matter. In the first case, a black line is used for protons, and a gray line is used for neutrons. 
The equation of state of hadronic matter in the presence of an external uniform magnetic field has been widely discussed. In particular, Ref. [15] makes an exhaustive analysis of the effects of the anomalous magnetic moments (AMMs) in matter composed of nucleons, light mesons, and electrons. One of the results shown there is the relevance of the AMMs for sufficiently high magnetic intensities. These results apparently contradict the conclusions of Ref. [28]. However, a direct comparison is not fair for several reasons: (i) A system of structureless charged fermions interacting solely with the electromagnetic field is considered in Ref. [28]. In such an approach, the AMMs are obtained from an expansion of the fermion self-energy. In hadronic physics instead, the AMMs are taken as constants determined mainly by the quark structure of hadrons. Hence, even neutral fermions exhibit nonzero AMMs. (ii) The equation of state is evaluated in Ref. [28] for only one species of charged fermions. This description can hardly be applied to realistic situations of hadronic physics. By way of illustration, neutron star matter can be considered. In such a case, the Coulomb repulsion among charged baryons is modified by the interaction with neutral ones. Furthermore, charged leptons are necessary to locally fulfill the requirement of charge neutrality. (iii) The explicit calculations of the equation of state shown in Ref. [28] are particularly misleading for hadronic physics, as the set of numerical values used there causes a loss of generality. For instance, the fermion mass fixed at $m=0.5 \mathrm{MeV}$ is almost irrelevant for baryons, and consequently the chemical potential $\mu=10 \mathrm{MeV} \simeq 0.05 \mathrm{fm}^{-1}$ corresponds, at zero temperature, to unphysical high densities (see Fig. 4).

\section{B. In-medium pionic mass}

The effective masses of the baryons and their chemical potentials obtained in the MFA are taken as input for the propagators (18)-(19) and (20)-(22). In turn, they are used to evaluate the polarization insertions (24) and (26) as functions of the density and the magnetic intensity.

In Figs. 5 and 6, the solutions of Eq. (27) are examined in terms of the particle density for several field intensities. The case $B=10^{19} \mathrm{G}$ is also included, because all the effects discussed are enlarged for this extremely strong field. It must be emphasized that we do not include vacuum corrections, and hadrons are regarded as elementary degrees of freedom.

The density dependence of the pion mass in neutral matter is exhibited in Fig. 5. The charged pions [Fig. 5(a)] receive contributions from $\Pi_{ \pm}^{\mathrm{WT}}$, and from the OPV between neutrons and protons in the Fermi sea. The first term does not depend explicitly on the magnetic intensity, but only through the particle density. Therefore, the same curve corresponds to different values of $B$ (dark lines). In fact, it can be shown that neglecting corrections from the OPV, the effective pion masses can be written as $m_{ \pm}^{*} / m_{\pi}=\sqrt{1+(a r)^{2}} \pm a r$, where $a=n_{0} / 4 f_{\pi}^{2} m_{\pi}$ and the relative density $r=n_{B} / n_{0}$ is introduced. When the

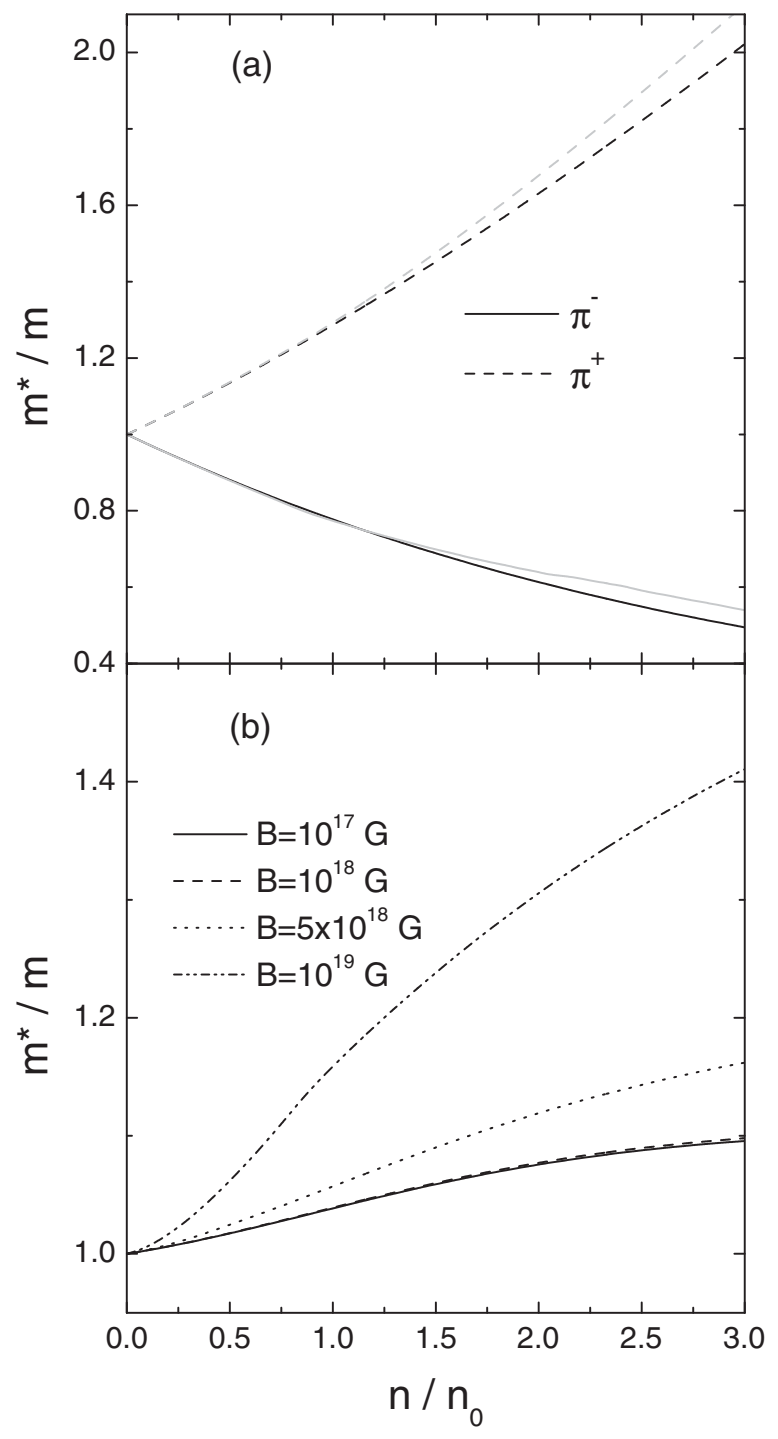

FIG. 5. The effective mass for (a) charged pions, and (b) the neutral pion, as a function of the density for several magnetic intensities in pure neutron matter.

full term is considered, a weak dependence on $B$ emerges. Only for the strongest intensity $B=10^{19} \mathrm{G}$ (gray lines) do the differences become appreciable.

The neutral pion receives a contribution only from the neutrons through $\Pi_{0}^{\mathrm{OPV}}$. The composition of this term is shown in detail in Eqs. (B2)-(B4). The factor $\Theta\left(\tilde{\mu}_{n}-\left|\mathcal{M}_{s}\right|\right)$ disfavors the spin-up contribution by reducing the integration domain of Eqs. (B3) and (B4), which numerically are both positive. As the spin polarization drops abruptly at low densities for $B=10^{17}-10^{18} \mathrm{G}$ [see Fig. 3(b)], $\Pi_{0}^{\mathrm{OPV}}$ partially loses the spin-up contribution at lower densities as compared to higher values of $B$. This explains the weaker growth at low and medium densities observed in Fig. 5(b) for $B<5 \times$ $10^{18} \mathrm{G}$. This effect is accentuated as the density increases, because $\tilde{\mu}_{n}$ shows a decreasing behavior (see Fig. 4). On the other hand, the coefficient $\kappa_{n} B$ in the second term of Eq. (B2) 
reduces drastically the contribution of Eq. (B4) when $B<5 \times 10^{18} \mathrm{G}$. From the numerical analysis, it is found that Eq. (B4) increases with density, and for a given value of $n_{B}$ is significantly greater than Eq. (B3). As a consequence, we observe a high slope for $B=10^{19} \mathrm{G}$, a moderate slope for $B=5 \times 10^{18} \mathrm{G}$, and a tiny slope for the remaining cases.

It must be pointed out that the dependence on the magnetic field shown in Fig. 5(b) is an exclusive consequence of the neutron magnetic moment. If $\kappa_{n}=0$, all the curves will coincide.

Symmetric nuclear matter is considered in Fig. 6; in such conditions, the polarization is solely due to the OPV. In particular, for the neutral pion [Fig. 6(c)], there is a sum of independent proton and neutron terms. The neutron contribution produces a smooth dependence, as discussed above. In fact, the results for the neutral pion mass in

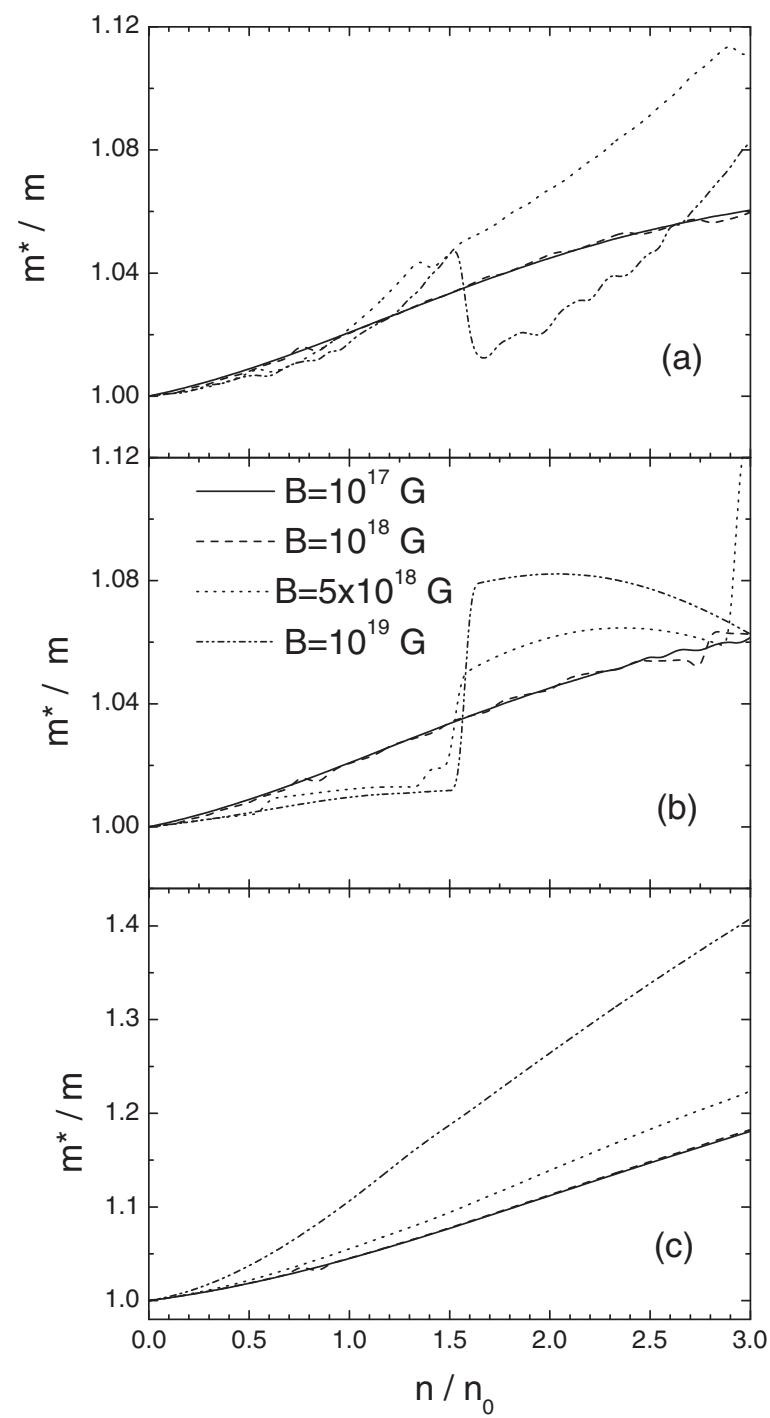

FIG. 6. The effective mass of (a) the negatively charged pion, (b) the positively charged pion, and (c) the neutral pion, as a function of the density for several magnetic intensities and isospin symmetric nuclear matter. neutron or symmetric nuclear matter are qualitatively similar. The rate of growth is slightly more pronounced for the latter case, with the exception of $B=10^{19} \mathrm{G}$.

For the charged pions, there is a mix of neutron and proton terms in each diagram. In contrast to the previous case, there are some pronounced irregularities, related to the occupation of the discrete Landau levels, which are emphasized as the magnetic field increases. The case of the negatively charged pion is examined in Fig. 6(a). For the lowest magnetic intensities, the proton levels are gradually and almost smoothly occupied as the density increases, reaching $n=$ 120 for $B=10^{17} \mathrm{G}$ and $n=12$ for $B=10^{18} \mathrm{G}$. As a consequence, the effective mass increases monotonously in the first case, and a mild oscillatory behavior appears in the second case. A drastic change is observed for $B=5 \times$ $10^{18} \mathrm{G}$. There are abrupt modifications of the slope at the densities $n_{B} / n_{0} \simeq 0.6,1.4,1.6$, and 2.9. To be more precise, the curve exhibits at these points local maxima followed by a fast increase. At these densities precisely, the opening of the Landau levels $n=1, s=1$ and $n=2, s=1$, the threshold of the proton depolarization [see Fig. 3(a)], and the beginning of the population of the $n=2, s=-1$ level, respectively, take place. In contrast to the previous cases, these changes take place at relatively greater densities (with higher Fermi momentum), hence the effect is amplified. For the most intense field considered here, $B=10^{19} \mathrm{G}$, a drop of roughly $4 \%$ occurs at $n_{B} / n_{0} \simeq 1.6$, followed by a further increase. At this point, the Landau level $n=1$ becomes available.

In fact, the same description, but at a considerably minor scale, holds for $B=5 \times 10^{18} \mathrm{G}, n_{B} / n_{0} \simeq 0.6$, where the proton phase is completely polarized and the population of the first excited Landau level is initiated. In regard to the positively charged pion, it must be noted that some pairs of terms in Eq. (B6) contribute with opposite signs, in contrast to the result for Eq. (B8). As a consequence, the same causes have qualitatively different manifestations for the results shown in Fig. 6(b), and for those just discussed for Fig. 6(a). For instance, when $B=10^{19} \mathrm{G}$, a jump in the effective mass is present at $n_{B} / n_{0} \simeq 1.6$. For densities above or below this point, the effective mass is increasing at the beginning, then it stabilizes and eventually decreases. This effect is due to the cancellation of pairs of terms growing with density but with opposite signs. A similar sketch is obtained for $B=5 \times 10^{18} \mathrm{G}$, but with significative points at $n_{B} / n_{0} \simeq 0.6,1.4,1.6,2.9$.

For the two remaining values of $B$, a smooth behavior is obtained, in agreement with the correspondent results shown in Fig. 6(a).

To complete this discussion, the effective pion mass obtained for symmetric nuclear matter in the lowest Landau level approximation is shown in Fig. 7. In this approach, the summation over Landau levels with $n \geq 1$ is neglected. From comparison with Fig. 6, we conclude that, with exception of the very low density regime, this approximation is not acceptable for intensities $B \leq 10^{18} \mathrm{G}$. As $B$ is 


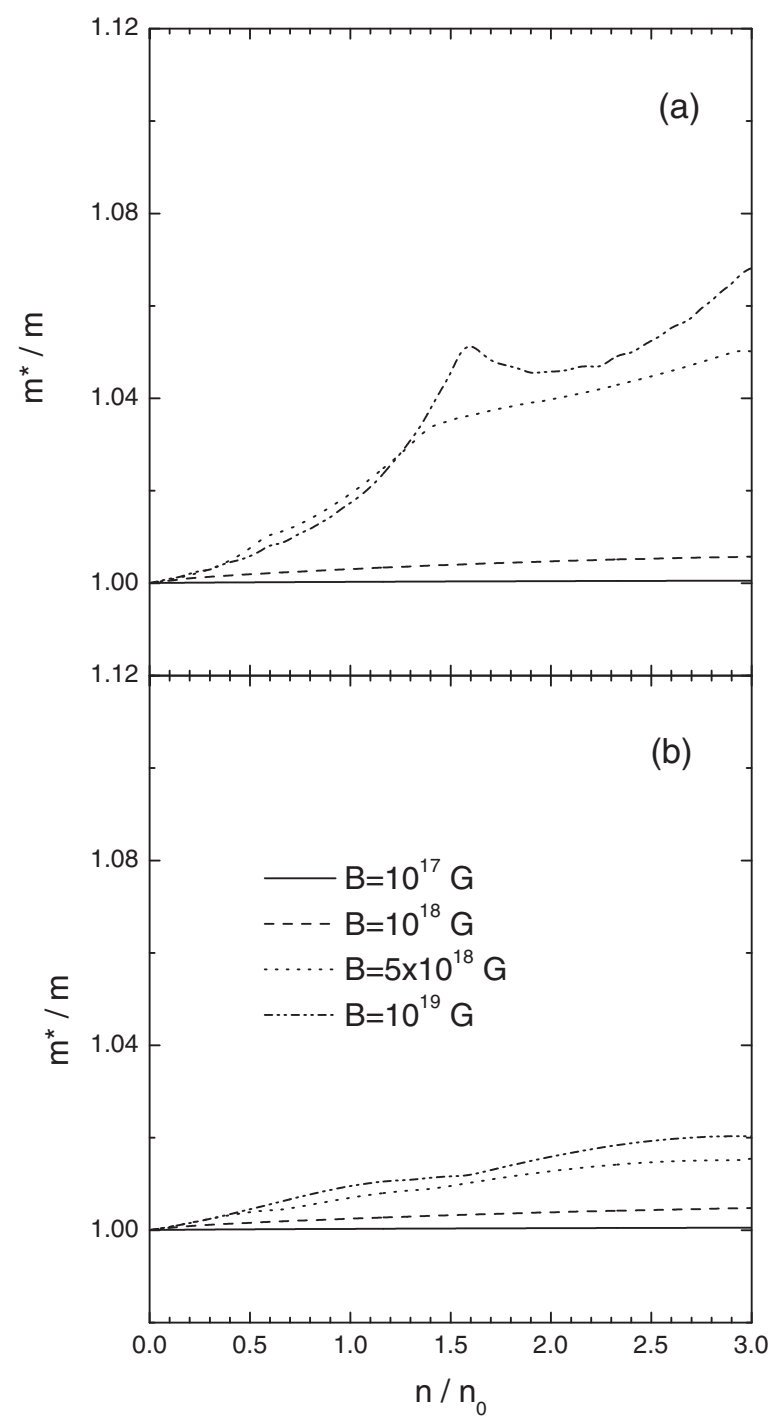

FIG. 7. The effective mass of the charged pions in the lowest Landau level approximation for symmetric nuclear matter as a function of the density. Results for (a) the negative pion and (b) the positive pion are shown for several magnetic intensities.

increased, the domain of the completely polarized proton phase is extended, and the approximation results are more adequate. For instance, qualitative agreement is obtained until $n_{B} / n_{0} \sim 1.5$ for $B=5 \times 10^{18}$ and $10^{19} \mathrm{G}$.

To end this section, we consider the imaginary part of the pion polarization, which is related to the in-medium dynamical stability of the particle. We have checked that the quantity $\tau=\left|\operatorname{Im} \Pi\left(p_{0}=m^{*}\right)\right| / m^{*}$ increases with the density and the magnetic intensity. For all the ranges of densities and magnetic intensities considered in our calculations, we have verified that $\tau<0.12$.

\section{CONCLUSIONS}

In this work, a covariant calculation of the propagator of Dirac and spin-zero Bose fields in the presence of a uniform external magnetic field has been presented. The nonzero magnetic moment of the fermion has been fully taken into account. The expressions found are valid for finite temperature and density. Furthermore, the gauge dependent contribution is reduced to a phase term as in the proper time evaluation of Ref. [17]. The propagators depend only on the four-momentum, improving the results found in Ref. [21].

The formalism has been applied to define an effective mass of the pion field, propagating in a dense nuclear medium at zero temperature. The approach proposed neglects divergent contributions from the Dirac sea, as is a common practice in QHD calculations. It must be taken into account that QHD models exhibit vacuum instabilities [29-31] which appear for transferred momentum above 2-3 GeV [29] when Dirac contributions to the diagram in Fig. 1(c) are included. This result questions the original interpretation of this kind of model as a realistic field theory description. A procedure for eliminating such instabilities was presented in Ref. [31]. The significant fact that the model breaks down at length scales $0.2 \mathrm{fm}$, smaller than the nucleon size, could indicate the emergence of substructure effects. Thus, QHD can be regarded as phenomenological descriptions which include interactions and solution procedures in its formulation. Therefore, it will be interesting to complement the results shown with a correction which takes account of the structure of hadrons.

The effective pion mass has been examined for two conditions of interest in practical application: pure neutron matter and isospin symmetric nuclear matter. Furthermore, we have focused on the domain of very strong magnetic fields, $B \geq 10^{17} \mathrm{G}$, and have covered particle densities below 3 times the nuclear saturation density.

In most situations, the effective mass increases with the density. As the magnetic intensity grows, the behavior is marked by the thresholds of the proton Landau levels and the change of the spin polarization of the nucleons.

Furthermore, by taking the imaginary part of the pion polarization as a measure of its stability in the nuclear medium, we have found that the neutral and positive pions are stable, and the negative pion becomes slightly unstable for high densities and magnetic intensities.

\section{ACKNOWLEDGMENTS}

This work has been partially supported by the Consejo Nacional de Investigaciones Cientficas y Tecnicas, Argentina.

\section{APPENDIX A: FOUR-MOMENTUM REPRESENTATION OF THE PROTON PROPAGATOR}

In order to derive Eqs. (20)-(22) from Eq. (17), we first integrate $p_{y}$ separately for the first and second terms between curly brackets.

In the first case, the relation 


$$
\int \frac{d p_{y}}{2 \pi} e^{i p_{y}\left(y^{\prime}-y\right)} e^{-\left(\xi^{2}+\xi^{2}\right) / 2}=\sqrt{\frac{q B}{4 \pi}} e^{i \Phi} e^{-q B\left[\left(x-x^{\prime}\right)^{2}+\left(y-y^{\prime}\right)^{2}\right] / 4}
$$

is used, which follows from Eq. 17.23 (13) of Ref. [27].

For the next step, the following relations will be useful:

$$
\int_{-\pi}^{\pi} d \theta e^{i z \cos (\theta-\varphi)}=2 \pi J_{0}(z)
$$

$$
\int_{-\pi}^{\pi} d \theta e^{ \pm i \theta} e^{i z \cos (\theta-\varphi)}=2 \pi i e^{ \pm i \varphi} J_{1}(z)
$$

which can be deduced with the help of Eq. 8.511 (4) of Ref. [27].

The last exponential on the right hand side of Eq. (A1) can be expressed in terms of a bidimensional integral on the momentum plane orthogonal to the external field:

$$
e^{-q B\left[\left(x-x^{\prime}\right)^{2}+\left(y-y^{\prime}\right)^{2}\right] / 4}=\frac{1}{\pi q B} \int_{0}^{\infty} d p_{\perp} p_{\perp} e^{-p_{\perp}^{2} / q B} \int_{-\pi}^{\pi} d \theta e^{i p_{\perp} R \cos (\theta-\varphi)},
$$

where $p_{\perp}=\sqrt{p_{x}^{2}+p_{y}^{2}}$, and $R=\sqrt{\left(x-x^{\prime}\right)^{2}+\left(y-y^{\prime}\right)^{2}}$. Furthermore, $\theta$, and $\varphi$ are the angular coordinates on the orthogonal planes of the vectors $\left(p_{x}, p_{y}\right)$ and $\left(x^{\prime}-x, y^{\prime}-y\right)$, respectively. For this purpose, Eq. 6.631 (4) of Ref. [27] and Eq. (A2) have been successively used. Thus, the relation

$$
\int \frac{d p_{y}}{2 \pi} e^{i p_{y}\left(y^{\prime}-y\right)} e^{-\left(\xi^{2}+\xi^{\prime 2}\right) / 2}=\sqrt{\frac{4 \pi}{q B}} e^{i \Phi} \int \frac{d p_{x} d p_{y}}{(2 \pi)^{2}} e^{i\left[p_{x}\left(x^{\prime}-x\right)+p_{y}\left(y^{\prime}-y\right)\right]} e^{-p_{\perp}^{2} / q B}
$$

is established.

On the other hand, by using Eq. 7.377 of Ref. [27], it can be shown that

$$
\begin{aligned}
\int \frac{d p_{y}}{2 \pi} e^{i p_{y}\left(y^{\prime}-y\right)} e^{-\left(\xi^{2}+\xi^{\prime 2}\right) / 2} \Lambda^{n s}= & \sqrt{\frac{q B}{4 \pi}} e^{i \Phi} e^{-q B R^{2} / 4} 2^{n} n !\left\{\left(u-\kappa B+s \Delta_{n}\right)\left[\Pi^{(+)} L_{n}+i \gamma_{1} \Pi^{(-)} \frac{m-s \Delta_{n}}{2 n} R e^{-i \varphi} L_{n-1}^{1}\right]\right. \\
& \left.+\left(\not{u}+\kappa B-s \Delta_{n}\right) \frac{s \Delta_{n}-m}{s \Delta_{n}+m}\left[\Pi^{(-)} L_{n-1}+i \gamma_{1} \Pi^{(+)} \frac{m+s \Delta_{n}}{2 n} \operatorname{Re}^{i \varphi} L_{n-1}^{1}\right]\right\},
\end{aligned}
$$

where the argument of all the Laguerre polynomials on the right-hand side is $q B R^{2} / 2$, and by definition $R \cos \varphi=x^{\prime}-x$, $R \sin \varphi=y^{\prime}-y$. Use has been made of the fact that Laguerre polynomials have definite parity.

Furthermore, the relations

$$
\begin{gathered}
e^{-q B R^{2} / 4} L_{n}\left(q B R^{2} / 2\right)=\frac{(-1)^{n}}{\pi q B} \int_{0}^{\infty} d p_{\perp} p_{\perp} e^{-p_{\perp}^{2} / q B} L_{n}\left(2 p_{\perp}^{2} / q B\right) \int_{-\pi}^{\pi} d \theta e^{i p_{\perp} R \cos (\theta-\varphi)}, \\
R e^{ \pm i \varphi} e^{-q B R^{2} / 4} L_{n}^{1}\left(q B R^{2} / 2\right)=\frac{(-1)^{n}}{2 \pi i}\left(\frac{2}{q B}\right)^{2} \int_{0}^{\infty} d p_{\perp} p_{\perp}^{2} e^{-p_{\perp}^{2} / q B} L_{n}^{1}\left(2 p_{\perp}^{2} / q B\right) \int_{-\pi}^{\pi} d \theta e^{ \pm i \theta} e^{i p_{\perp} R \cos (\theta-\varphi)}
\end{gathered}
$$

are obtained from Eq. 7.421 (4) of Ref. [27] and Eqs. (A2)-(A3).

When the last two equations are inserted into Eq. (A4), the result

$$
\begin{aligned}
\int \frac{d p_{y}}{2 \pi} e^{i p_{y}\left(y^{\prime}-y\right)} e^{-\left(\xi^{2}+\xi^{\prime 2}\right) / 2} \Lambda^{n s}= & \sqrt{\frac{4 \pi}{q B}} e^{i \Phi} 2^{n} n !(-1)^{n} \int \frac{d p_{x} d p_{y}}{(2 \pi)^{2}} e^{i\left[p_{x}\left(x^{\prime}-x\right)+p_{y}\left(y^{\prime}-y\right)\right]} e^{-p_{\perp}^{2} / q B} \\
& \times\left\{\left(\not{u}-\kappa B+s \Delta_{n}\right) \Pi^{(+)} L_{n}-\left(\not{u}+\kappa B-s \Delta_{n}\right) \Pi^{(-)} \frac{s \Delta_{n}-m}{s \Delta_{n}+m} L_{n-1}\right. \\
& \left.+\frac{s \Delta_{n}-m}{n q B} p_{\perp}\left[\left(\not{u}-\kappa B+s \Delta_{n}\right) \gamma^{1} \Pi^{(-)} e^{-i \theta}-\left(\not{u}+\kappa B-s \Delta_{n}\right) \gamma^{1} \Pi^{(+)} e^{i \theta}\right] L_{n-1}^{1}\right\}
\end{aligned}
$$

is obtained. 
After some algebra on the last term between curly brackets and the use of Eq. 8.971 (4) of Ref. [27] to put $L_{k}^{(1)}$ in terms of $L_{k}-L_{k-1}$, we obtain the final expression:

$$
\begin{aligned}
\int \frac{d p_{y}}{2 \pi} e^{i p_{y}\left(y^{\prime}-y\right)} e^{-\left(\xi^{2}+\xi^{\prime 2}\right) / 2} \Lambda^{n s}= & \sqrt{\frac{4 \pi}{q B}} e^{i \Phi} 2^{n} n !(-1)^{n} \int \frac{d p_{x} d p_{y}}{(2 \pi)^{2}} e^{i\left[p_{x}\left(x^{\prime}-x\right)+p_{y}\left(y^{\prime}-y\right)\right]} e^{-p_{\perp}^{2} / q B} \\
& \times\left\{\left(u-\kappa B+s \Delta_{n}\right) \Pi^{(+)} L_{n}-\left(u+\kappa B-s \Delta_{n}\right) \Pi^{(-)} \frac{s \Delta_{n}-m}{s \Delta_{n}+m} L_{n-1}\right. \\
& \left.+\frac{s \Delta_{n}-m}{2 p_{\perp}^{2}}\left[u+\left(s \Delta_{n}-\kappa B\right) i \gamma^{1} \gamma^{2}\right] i \gamma^{1} \gamma^{2} \not v\left(L_{n}-L_{n-1}\right)\right\} .
\end{aligned}
$$

\section{APPENDIX B: PION POLARIZATION FUNCTION FOR TIME-LIKE MOMENTUM}

Here we give explicit formulas for the one-pion exchange contribution to the pion polarization at $\mathbf{p}=0$.

Since

$$
\Pi_{0}=\Pi_{0}^{(n)}+\Pi_{0}^{(p)}
$$

we have

$$
\begin{aligned}
\operatorname{Re} \Pi_{0}^{(n)}\left(p_{0}\right)= & \left(\frac{g_{A}}{4 \pi f_{\pi}}\right)^{2} \int_{0}^{\infty} \frac{d t}{\Delta^{2}} \sum_{s, s^{\prime}}\left[2 m^{2}+\left(s s^{\prime}-1\right) \Delta^{2}\right] \Theta\left(\tilde{\mu}_{n}-\left|\mathcal{M}_{s}\right|\right)\left(\mathcal{M}_{s}+\mathcal{M}_{s^{\prime}}\right) \\
& \times\left\{\left(\mathcal{M}_{s^{\prime}}-\mathcal{M}_{s}\right) \log \left(\frac{\tilde{\mu}_{n}+p_{F s}}{\tilde{\mu}_{n}-p_{F s}}\right)+\eta\left(\mathcal{M}_{s}+\mathcal{M}_{s^{\prime}}\right) \frac{p_{0}^{2}-\left(\mathcal{M}_{s}-\mathcal{M}_{s^{\prime}}\right)^{2}}{\Lambda}\right. \\
& \times\left[2 \Theta\left(4 p_{0}^{2} \mathcal{M}_{s}^{2}-\left(p_{0}^{2}+\mathcal{M}_{s}^{2}-\mathcal{M}_{s^{\prime}}^{2}\right)^{2}\right) \arctan \left(\frac{p_{0}^{2}+\mathcal{M}_{s}^{2}-\mathcal{M}_{s^{\prime}}^{2}}{\eta \Lambda \tilde{\mu}_{n}} p_{F s}\right)\right. \\
& \left.\left.-\Theta\left(\left(p_{0}^{2}+\mathcal{M}_{s}^{2}-\mathcal{M}_{s^{\prime}}^{2}\right)^{2}-4 p_{0}^{2} \mathcal{M}_{s}^{2}\right) \log \left(\frac{\left.\Lambda \tilde{\mu}_{n}+\eta\left(p_{0}^{2}+\mathcal{M}_{s}^{2}-\mathcal{M}_{s^{\prime}}^{2}\right) p_{F s}\right)}{\left.\Lambda \tilde{\mu}_{n}-\eta\left(p_{0}^{2}+\mathcal{M}_{s}^{2}-\mathcal{M}_{s^{\prime}}^{2}\right) p_{F s}\right)}\right)\right]\right\},
\end{aligned}
$$

where $\Delta=\sqrt{m^{2}+t}, \mathcal{M}_{s}=s \Delta-\kappa_{n} B, p_{F s}=\sqrt{\tilde{\mu}_{n}^{2}-\mathcal{M}_{s}^{2}}, \eta=\operatorname{sgn}\left(p_{0}\right)$, and $\Lambda=\sqrt{\mid 4 p_{0}^{2} \mathcal{M}_{s}^{2}-\left(p_{0}^{2}+\mathcal{M}_{s}^{2}-\mathcal{M}_{s^{\prime}}^{2}\right)^{2}}$. Although the domain of integration is not bounded, the relation

$$
\Theta\left(\tilde{\mu}_{n}-\left|\mathcal{M}_{s}\right|\right) \equiv \Theta\left(\left(\tilde{\mu}_{n}+s \kappa_{n} B\right)^{2}-m^{2}-t\right) \Theta\left(\tilde{\mu}_{n}+s \kappa_{n} B-m\right),
$$

which is valid for the conditions under consideration, establishes an upper limit of integration. By performing the sum over $s^{\prime}$, it can be rewritten as

$$
\begin{gathered}
\operatorname{Re}_{0}^{(n)}\left(p_{0}\right)=\left(\frac{g_{A}}{\pi f_{\pi}}\right)^{2} \int_{0}^{\infty} \frac{d t}{2 \Delta^{2}} \sum_{s} \Theta\left(\tilde{\mu}_{n}-\left|\mathcal{M}_{s}\right|\right)\left(A_{1 s}-\kappa_{n} B t A_{2 s}\right), \\
A_{1 s}=p_{0} m^{2} \frac{\mathcal{M}_{s}^{2}}{\lambda}\left[2 \Theta\left(4 \mathcal{M}_{s}^{2}-p_{0}^{2}\right) \arctan \left(\frac{p_{0} p_{F s}}{\tilde{\mu}_{n} \lambda}\right)+\Theta\left(p_{0}^{2}-4 \mathcal{M}_{s}^{2}\right) \log \left(\frac{\tilde{\mu}_{n} \lambda-p_{0} p_{F s}}{\tilde{\mu}_{n} \lambda+p_{0} p_{F s}}\right)\right], \\
A_{2 s}=s \Delta \log \left(\frac{\tilde{\mu}_{n}+p_{F s}}{\tilde{\mu}_{n}-p_{F s}}\right)+\eta \kappa_{n} B \frac{p_{0}^{2}-4 \Delta}{\lambda^{\prime}}\left[2 \Theta\left(4 p_{0}^{2} \mathcal{M}_{s}^{2}-\left(p_{0}^{2}-4 s \kappa_{n} B \Delta\right)^{2}\right) \arctan \left(\eta p_{F s} \frac{p_{0}^{2}-s \kappa_{n} B \Delta}{\tilde{\mu}_{n} \lambda^{\prime}}\right)\right. \\
\left.+\Theta\left(\left(p_{0}^{2}-4 s \kappa_{n} B \Delta\right)^{2}-4 p_{0}^{2} \mathcal{M}_{s}^{2}\right) \log \left(\frac{\tilde{\mu}_{n} \lambda^{\prime}-\eta\left(p_{0}^{2}-4 s \kappa_{n} B \Delta_{2}\right) p_{F s}}{\tilde{\mu}_{n} \lambda^{\prime}+\eta\left(p_{0}^{2}-4 s \kappa_{n} B \Delta_{2}\right) p_{F s}}\right)\right],
\end{gathered}
$$

where $\lambda=\sqrt{\left|p_{0}^{2}-4 \mathcal{M}_{s}^{2}\right|}$, and $\lambda^{\prime}=\sqrt{\left|4 p_{0}^{2} \mathcal{M}_{s}^{2}-\left(p_{0}^{2}+4 s \kappa_{n} B \Delta_{2}\right)^{2}\right|}$. 


$$
\begin{aligned}
& \operatorname{Im} \Pi_{0}^{(n)}\left(p_{0}\right)=\left(\frac{g_{A}}{4 f_{\pi}}\right)^{2} \int_{0}^{\infty} \frac{d t}{\pi} \sum_{s, s^{\prime}} \frac{s s^{\prime}}{\Delta^{2}}\left[2 m^{2}+\left(s s^{\prime}-1\right) \Delta^{2}\right] \frac{\left(\mathcal{M}_{s}+\mathcal{M}_{s^{\prime}}\right)^{2}}{\Lambda_{2}}\left[p_{0}^{2}-\left(\mathcal{M}_{s}-\mathcal{M}_{s^{\prime}}\right)^{2}\right] \\
& \times\left\{\Theta\left(\tilde{\mu}_{n}-\frac{\mathcal{M}_{s}^{2}-\mathcal{M}_{s^{\prime}}^{2}+p_{0}^{2}}{2 p_{0}}\right) \Theta\left(\frac{\left(p_{0}-\left|\mathcal{M}_{s}\right|\right)^{2}-\mathcal{M}_{s^{\prime}}^{2}}{2 p_{0}}\right)\left[2 \Theta\left(\tilde{\mu}_{n}-\frac{\mathcal{M}_{s}^{2}-\mathcal{M}_{s^{\prime}}^{2}-p_{0}^{2}}{2 p_{0}}\right)-1\right]\right. \\
& \left.+\Theta\left(\tilde{\mu}_{n}-\frac{\mathcal{M}_{s}^{2}-\mathcal{M}_{s^{\prime}}^{2}-p_{0}^{2}}{2 p_{0}}\right) \Theta\left(\frac{\mathcal{M}_{s}^{2}-\left(p_{0}^{2}+\left|\mathcal{M}_{s^{\prime}}\right|\right)^{2}}{2 p_{0}}\right)\right\}, \\
& \operatorname{Re} \Pi_{0}^{(p)}\left(p_{0}\right)=\left(\frac{g_{A}}{\pi f_{\pi}}\right)^{2} q B\left[\Theta\left(\tilde{\mu}_{p}-\left|\mathcal{M}_{0}\right|\right) A_{0}+\frac{1}{16} \sum_{n, s, s^{\prime}} \Theta\left(\tilde{\mu}_{p}-\left|\mathcal{M}_{n s}\right|\right) A_{n s s^{\prime}}\right], \\
& A_{0}=p_{0} \frac{\mathcal{M}_{0}^{2}}{\lambda_{0}}\left[2 \Theta\left(4 \mathcal{M}_{0}^{2}-p_{0}^{2}\right) \arctan \left(\frac{p_{0} p_{F 0}}{\tilde{\mu}_{p} \lambda_{0}}\right)+\Theta\left(p_{0}^{2}-4 \mathcal{M}_{0}^{2}\right) \log \left(\frac{\tilde{\mu}_{p} \lambda_{0}-p_{0} p_{F 0}}{\tilde{\mu}_{p} \lambda_{0}+p_{0} p_{F 0}}\right)\right], \\
& A_{n s s^{\prime}}=\frac{\Delta_{n}+s m}{\Delta_{n}} \frac{\Delta_{n}+s^{\prime} m}{\Delta_{n}}\left(1-2 \frac{m-s^{\prime} \Delta_{n}}{m+s \Delta_{n}}+\frac{m-s \Delta_{n}}{m+s \Delta_{n}} \frac{m-s^{\prime} \Delta_{n}}{m+s^{\prime} \Delta_{n}}\right) \\
& \times\left\{\left(\mathcal{M}_{n s^{\prime}}^{2}-\mathcal{M}_{n s^{\prime}}^{2}\right) \log \left(\frac{\tilde{\mu}_{p}+p_{F n s}}{\tilde{\mu}_{p}-p_{F n s}}\right)+\frac{\eta}{\Lambda_{1}}\left(\mathcal{M}_{n s}+\mathcal{M}_{n s^{\prime}}\right)^{2}\left[p_{0}^{2}-\left(\mathcal{M}_{n s}-\mathcal{M}_{n s^{\prime}}\right)^{2}\right]\right. \\
& \times\left[2 \Theta\left(4 p_{0}^{2} \mathcal{M}_{n s}^{2}-\left(p_{0}^{2}+\mathcal{M}_{n s}^{2}-\mathcal{M}_{n s^{\prime}}^{2}\right)^{2}\right) \arctan \left(\eta p_{F n s} \frac{p_{0}^{2}+\mathcal{M}_{n s}^{2}-\mathcal{M}_{n s^{\prime}}^{2}}{\tilde{\mu}_{p} \Lambda_{1}}\right)\right. \\
& \left.\left.+\Theta\left(\left(p_{0}^{2}+\mathcal{M}_{n s}^{2}-\mathcal{M}_{n s^{\prime}}^{2}\right)^{2}-4 p_{0}^{2} \mathcal{M}_{n s}^{2}\right) \log \left(\frac{\tilde{\mu}_{p} \Lambda_{1}-\eta\left(p_{0}^{2}+\mathcal{M}_{n s}^{2}-\mathcal{M}_{n s^{\prime}}^{2}\right) p_{F n s}}{\tilde{\mu}_{p} \Lambda_{1}+\eta\left(p_{0}^{2}+\mathcal{M}_{n s}^{2}-\mathcal{M}_{n s^{\prime}}^{2}\right) p_{F n s}}\right),\right]\right\}
\end{aligned}
$$

with $\quad \mathcal{M}_{0}=m-\kappa_{p} B, \quad \mathcal{M}_{n s}=s \Delta_{n}-\kappa_{p} B, \quad p_{F 0}=\sqrt{\tilde{\mu}_{p}^{2}-\mathcal{M}_{0}^{2}}, \quad p_{F n s}=\sqrt{\tilde{\mu}_{p}^{2}-\mathcal{M}_{n s}^{2}}, \quad \lambda_{0}=\sqrt{\left|p_{0}^{2}-4 \mathcal{M}_{0}^{2}\right|}, \quad$ and $\Lambda_{1}=\sqrt{\left|4 p_{0}^{2} \mathcal{M}_{n s}^{2}-\left(p_{0}^{2}+\mathcal{M}_{n s}^{2}-\mathcal{M}_{n s^{\prime}}^{2}\right)^{2}\right|}$

$$
\begin{aligned}
\operatorname{Im} \Pi_{0}^{(p)}\left(p_{0}\right)= & \left(\frac{g_{A}}{f_{\pi}}\right)^{2} \frac{q B}{2 \pi}\left(C_{0}+\frac{1}{8} \sum_{n s s^{\prime}} C_{n s s^{\prime}}\right) \\
C_{0}= & 2 \eta p_{0} \frac{\mathcal{M}_{0}^{2}}{\lambda_{0}}\left\{\Theta\left(-p_{0}-2 \mathcal{M}_{0}\right) \Theta\left(\tilde{\mu}_{p}+p_{0} / 2\right)+\Theta\left(p_{0}-2 \mathcal{M}_{0}\right) \Theta\left(\tilde{\mu}_{p}-p_{0} / 2\right)\left[1-2 \Theta\left(\tilde{\mu}_{p}+p_{0} / 2\right)\right]\right\} \\
C_{n s s^{\prime}}= & \left(\Delta_{n}+s m\right)\left(\Delta_{n}+s^{\prime} m\right)\left(\frac{\mathcal{M}_{n s}-\mathcal{M}_{n s^{\prime}}}{\Delta_{n}}\right)^{2} \frac{p_{0}^{2}-\left(\mathcal{M}_{n s}-\mathcal{M}_{n s^{\prime}}\right)^{2}}{\Lambda_{1}}\left(1-2 \frac{m-s^{\prime} \Delta_{n}}{m+s \Delta_{n}}+\frac{m-s \Delta_{n}}{m+s \Delta_{n}} \frac{m+s^{\prime} \Delta_{n}}{m+s^{\prime} \Delta_{n}}\right) \\
& \times\left\{\Theta\left(\frac{\left(p_{0}-\mathcal{M}_{n s}\right)^{2}-\mathcal{M}_{n s^{\prime}}^{2}}{2 p_{0}}\right) \Theta\left(\tilde{\mu}_{p}-\frac{p_{0}^{2}+\mathcal{M}_{n s}^{2}-\mathcal{M}_{n s^{\prime}}^{2}}{2 p_{0}}\right)\left[1-2 \Theta\left(\tilde{\mu}_{p}+\frac{\left.\left.p_{0}^{2}+\mathcal{M}_{n s^{\prime}}^{2}-\mathcal{M}_{n s}^{2}\right)\right]}{2 p_{0}}\right)\right]\right. \\
& \left.+\Theta\left(\frac{\mathcal{M}_{n s}^{2}-\left(p_{0}+\mathcal{M}_{n s^{\prime}}\right)^{2}}{2 p_{0}}\right) \Theta\left(\tilde{\mu}_{p}+\frac{p_{0}^{2}+\mathcal{M}_{n s^{\prime}}^{2}-\mathcal{M}_{n s}^{2}}{2 p_{0}}\right)\right\} \\
\operatorname{Re}_{+}\left(p_{0}\right)= & \left(\frac{g_{A}}{4 \pi f_{\pi}}\right)^{2} \int_{0}^{\infty} \frac{d t}{\Delta} e^{-t / q B} \sum_{s^{\prime}}\left\{\left(\Delta+s^{\prime} m\right)\left[\Theta\left(\tilde{\mu}_{p}-\left|\mathcal{M}_{0}\right|\right) F_{p}\left(p_{F 0}, \mathcal{M}_{0}, \lambda_{0}\right)+\Theta\left(\tilde{\mu}_{n}-\left|\mathcal{M}_{s^{\prime}}\right|\right) F_{n}\left(p_{F 0}, \mathcal{M}_{0}, \lambda_{0}\right)\right]\right. \\
& +\sum_{n s}(-1)^{n} \frac{\Delta_{n}+s m}{2 \Delta_{n}}\left[\left(\Delta+s^{\prime} m\right) L_{n}-\left(\Delta-s^{\prime} m\right) \frac{s \Delta_{n}-m}{s \Delta_{n}+m} L_{n-1}-s^{\prime}\left(s \Delta_{n}-m\right)\left(L_{n}-L_{n-1}\right)\right] \\
& \left.\times\left[\Theta\left(\tilde{\mu}_{p}-\left|\mathcal{M}_{n s}\right|\right) F_{p}\left(p_{F n s}, \mathcal{M}_{n s}, \Lambda_{1}\right)+\Theta\left(\tilde{\mu}_{n}-\left|\mathcal{M}_{s^{\prime}}\right|\right) F_{n}\left(p_{F s^{\prime}}, \mathcal{M}_{n s}, \Lambda_{1}\right)\right]\right\}
\end{aligned}
$$




$$
\begin{aligned}
F_{a}(x, M, U)= & -I_{a}\left[4 p_{0} x+\left(M^{2}-\mathcal{M}_{s^{\prime}}^{2}\right) \log \left(\frac{\tilde{\mu}_{a}+x}{\tilde{\mu}_{a}-x}\right)\right]+\frac{\eta}{U}\left(\mathcal{M}_{s^{\prime}}+M\right)^{2}\left[p_{0}^{2}-\left(\mathcal{M}_{s^{\prime}}-M\right)^{2}\right] \\
& \times\left\{2 \Theta\left(4 p_{0}^{2} M^{2}-\left(p_{0}^{2}+M^{2}-\mathcal{M}_{s^{\prime}}^{2}\right)^{2}\right)\left[\arctan \left(\eta x \frac{p_{0}^{2}+I_{a}\left(M^{2}-\mathcal{M}_{s^{\prime}}^{2}\right)}{\tilde{\mu}_{a} U}\right)+I_{a} \arctan \left(2 \eta p_{0} \frac{x}{U}\right)\right]\right. \\
& \left.+\Theta\left(\left(p_{0}^{2}+M^{2}-\mathcal{M}_{s^{\prime}}^{2}\right)^{2}-4 p_{0}^{2} M^{2}\right) I_{a}\left[\log \left(\frac{\tilde{\mu}_{a} U-\eta\left(p_{0}^{2} I_{a}+M^{2}-\mathcal{M}_{s^{\prime}}^{2}\right) x}{\tilde{\mu}_{a} U+\eta\left(p_{0}^{2} I_{a}+M^{2}-\mathcal{M}_{s^{\prime}}^{2} x\right.}\right)+\log \left(\frac{U-2 \eta p_{0} x}{U+2 \eta p_{0} x}\right)\right]\right\},
\end{aligned}
$$

where we have introduced the isospin projection number $I_{p}=1, I_{n}=-1$, and all Laguerre functions have the same argument $L_{k}(2 t / q B)$.

$$
\begin{aligned}
& \operatorname{Im} \Pi_{+}\left(p_{0}\right)=\left(\frac{g_{A}}{4 f_{\pi}}\right)^{2} \int_{0}^{\infty} \frac{d t}{\pi} \frac{e^{-t / q B}}{\Delta} \sum_{s^{\prime}}\left\{2\left(\Delta+s^{\prime} m\right) G\left(\mathcal{M}_{0}, \lambda_{0}\right)+\sum_{n s}(-1)^{n} \frac{\Delta_{n}+s m}{\Delta_{n}}\right. \\
& \left.\times\left[\left(\Delta+s^{\prime} m\right) L_{n}-\left(\Delta-s^{\prime} m\right) \frac{s \Delta_{n}-m}{s \Delta_{n}+m} L_{n-1}-s^{\prime}\left(s \Delta_{n}-m\right)\left(L_{n}-L_{n-1}\right)\right] G\left(\mathcal{M}_{n s}, \Lambda_{1}\right)\right\}, \\
& G(M, U)=\left(\mathcal{M}_{s^{\prime}}+M\right)^{2} \frac{p_{0}^{2}-\left(\mathcal{M}_{s^{\prime}}-M\right)^{2}}{U}\left\{\Theta\left(\frac{M^{2}-\left(p_{0}+\left|\mathcal{M}_{s^{\prime}}\right|\right)^{2}}{2 p_{0}}\right) \Theta\left(\tilde{\mu}_{n}-\frac{M^{2}-\mathcal{M}_{s^{\prime}}^{2}-p_{0}^{2}}{2 p_{0}}\right)\right. \\
& \left.+\Theta\left(\frac{\left(p_{0}-|M|\right)^{2}-\mathcal{M}_{s^{\prime}}^{2}}{2 p_{0}}\right) \Theta\left(\tilde{\mu}_{p}-\frac{M^{2}-\mathcal{M}_{s^{\prime}}^{2}+p_{0}^{2}}{2 p_{0}}\right)\left[1-2 \Theta\left(\tilde{\mu}_{n}-\frac{M^{2}-\mathcal{M}_{s^{\prime}}^{2}-p_{0}^{2}}{2 p_{0}}\right)\right]\right\}, \\
& \operatorname{Re}_{-}\left(p_{0}\right)=\left(\frac{g_{A}}{4 \pi f_{\pi}}\right)^{2} \int_{0}^{\infty} \frac{d t}{\Delta} e^{-t / q B} \sum_{s^{\prime}}\left\{( \Delta + s ^ { \prime } m ) \left[\Theta\left(\tilde{\mu}_{p}-\left|\mathcal{M}_{0}\right|\right) G_{p}\left(p_{F 0}, \mathcal{M}_{0}, \lambda_{0}\right)\right.\right. \\
& \left.+\Theta\left(\tilde{\mu}_{n}-\left|\mathcal{M}_{s^{\prime}}\right|\right) G_{n}\left(p_{F 0}, \mathcal{M}_{0}, \lambda_{0}\right)\right]+\sum_{n s}(-1)^{n} \frac{\Delta_{n}+s m}{2 \Delta_{n}} \\
& \times\left[\left(\Delta+s^{\prime} m\right) L_{n}-\left(\Delta-s^{\prime} m\right) \frac{s \Delta_{n}-m}{s \Delta_{n}+m} L_{n-1}-s^{\prime}\left(s \Delta_{n}-m\right)\left(L_{n}-L_{n-1}\right)\right] \\
& \left.\times\left[\Theta\left(\tilde{\mu}_{p}-\left|\mathcal{M}_{n s}\right|\right) G_{p}\left(p_{F n s}, \mathcal{M}_{n s}, \Lambda_{1}\right)+\Theta\left(\tilde{\mu}_{n}-\left|\mathcal{M}_{s^{\prime}}\right|\right) G_{n}\left(p_{F s^{\prime}}, \mathcal{M}_{n s}, \Lambda_{1}\right)\right]\right\}, \\
& G_{a}(x, M, U)=I_{a}\left[4 p_{0} x+\left(\mathcal{M}_{s^{\prime}}^{2}-M^{2}\right) \log \left(\frac{\tilde{\mu}_{a}+x}{\tilde{\mu}_{a}-x}\right)\right]+\frac{\eta}{U}\left(\mathcal{M}_{s^{\prime}}+M\right)^{2}\left[p_{0}^{2}-\left(\mathcal{M}_{s^{\prime}}-M\right)^{2}\right] \\
& \times\left\{2 \Theta ( 4 p _ { 0 } ^ { 2 } M ^ { 2 } - ( p _ { 0 } ^ { 2 } + M ^ { 2 } - \mathcal { M } _ { s ^ { \prime } } ^ { 2 } ) ^ { 2 } ) \left[\arctan \left(\eta x \frac{p_{0}^{2}+I_{a}\left(M^{2}-\mathcal{M}_{s^{\prime}}^{2}\right)}{\tilde{\mu}_{a} U}\right)\right.\right. \\
& \left.-I_{a} \arctan \left(2 \eta p_{0} \frac{x}{U}\right)\right]+\Theta\left(\left(p_{0}^{2}+M^{2}-\mathcal{M}_{s^{\prime}}^{2}\right)^{2}-4 p_{0}^{2} M^{2}\right) I_{a} \\
& \left.\times\left[\log \left(\frac{\tilde{\mu}_{a} U-\eta\left(p_{0}^{2} I_{a}+M^{2}-\mathcal{M}_{s^{\prime}}^{2}\right) x}{\tilde{\mu}_{a} U+\eta\left(p_{0}^{2} I_{a}+M^{2}-\mathcal{M}_{s^{\prime}}^{2} x\right.}\right)+\log \left(\frac{U+2 \eta p_{0} x}{U-2 \eta p_{0} x}\right)\right]\right\}, \\
& \operatorname{Im} \Pi_{-}\left(p_{0}\right)=\left(\frac{g_{A}}{4 f_{\pi}}\right)^{2} \int_{0}^{\infty} \frac{d t}{\pi} \frac{e^{-t / q B}}{\Delta} \sum_{s^{\prime}}\left\{2\left(\Delta+s^{\prime} m\right) H\left(\mathcal{M}_{0}, \lambda_{0}\right)+\sum_{n s}(-1)^{n} \frac{\Delta_{n}+s m}{\Delta_{n}}\right. \\
& \left.\left.\times\left[\left(\Delta+s^{\prime} m\right) L_{n}-\left(\Delta-s^{\prime} m\right) \frac{s \Delta_{n}-m}{s \Delta_{n}+m} L_{n-1}-s^{\prime}\left(s \Delta_{n}-m\right)\left(L_{n}-L_{n-1}\right)\right] H\left(\mathcal{M}_{n s}, \Lambda_{1}\right)\right]\right\}, \\
& H(M, U)=\left(\mathcal{M}_{s^{\prime}}+M\right)^{2} \frac{p_{0}^{2}-\left(\mathcal{M}_{s^{\prime}}-M\right)^{2}}{U}\left\{\Theta\left(\frac{\mathcal{M}_{s^{\prime}}^{2}-\left(p_{0}+|M|\right)^{2}}{2 p_{0}}\right) \Theta\left(\tilde{\mu}_{p}-\frac{\mathcal{M}_{s^{\prime}}^{2}-M^{2}-p_{0}^{2}}{2 p_{0}}\right)\right. \\
& \left.+\Theta\left(\frac{\left(p_{0}-\left|\mathcal{M}_{s^{\prime}}\right|\right)^{2}-M^{2}}{2 p_{0}}\right) \Theta\left(\tilde{\mu}_{n}-\frac{\mathcal{M}_{s^{\prime}}^{2}-M^{2}+p_{0}^{2}}{2 p_{0}}\right)\left[1-2 \Theta\left(\tilde{\mu}_{p}-\frac{\mathcal{M}_{s^{\prime}}^{2}-M^{2}-p_{0}^{2}}{2 p_{0}}\right)\right]\right\} .
\end{aligned}
$$

The expressions for the pion polarizations $\Pi_{-}^{\mathrm{OPV}}$ and $\Pi_{+}^{\mathrm{OPV}}$ are formally related by the simple transformation $p_{0} \rightarrow-p_{0}$. 
[1] D. Lai, Rev. Mod. Phys. 73, 629 (2001).

[2] V. A. Miransky and I. A. Shovkovy, Phys. Rep. 576, 1 (2015).

[3] J. Dong, U. Lombardo, W. Zuo, and H. Zhang, Nucl. Phys. A898, 32 (2013).

[4] J. Dong, W. Zuo, and J. Gu, Phys. Rev. D 87, 103010 (2013).

[5] R. Aguirre, E. Bauer, and I. Vidaña, Phys. Rev. C 89, 035809 (2014).

[6] A. Rabhi, M. A. Perez-Garcia, C. Providencia, and I. Vidana, Phys. Rev. C 91, 045803 (2015).

[7] Z. Rezaei and G. H. Bordbar, Eur. Phys. J. A 52, 132 (2016).

[8] D. E. Kharzeev, Phys. Lett. B 633, 260 (2006); D. E. Kharzeev, L. D. McLerran, and H. J. Warringa, Nucl. Phys. A803, 227 (2008).

[9] Y.-J. Mo, S.-Q. Feng, and Y.-F. Shi, Phys. Rev. C 88, 024901 (2013).

[10] V. V. Skokov, A. Y. Illarionov, and V. D. Toneev, Int. J. Mod. Phys. A 24, 5925 (2009).

[11] R. C. Duncan and C. Thompson, Astrophys. J. 392, L9 (1992).

[12] C. Thompson and R. C. Duncan, Mon. Not. Roy. Astron. Soc. 275, 255 (1995); Astrophys. J. 473, 322 (1996).

[13] B. D. Serot and J. D. Walecka, Adv. Nucl. Phys. 16, 1 (1986).

[14] S. Chakrabarty, D. Bandyopadhyay, and S. Pal, Phys. Rev. Lett. 78, 2898 (1997).

[15] A. Broderick, M. Prakash, and J. M. Lattimer, Astrophys. J. 537, 351 (2000).
[16] R. Mallick, R. Gopal, S. J. Ghosh, S. Raha, and S. Roychowdhury, J. Phys. G 39, 095201 (2012).

[17] J. Schwinger, Phys. Rev. 82, 664 (1951).

[18] V. I. Ritus, Sov. Phys. JETP 48, 788 (1978).

[19] T. Herpay and A. Patkos, J. Phys. G 35, 025201 (2008).

[20] A. V. Kuznetsov and A. A. Okrugin, Int. J. Mod. Phys. A 26, 2725 (2011).

[21] R. M. Aguirre and A. L. De Paoli, Eur. Phys. J. A 52, 343 (2016).

[22] A. Ayala, A. Bashir, A. Raya, and A. Sanchez, J. Phys. G 37, 015001 (2010).

[23] T. Maruyama, M. K. Cheoun, T. Kajino, Y. Kwon, G. J. Mathews, and C. Y. Ryu, Phys. Rev. D 91, 123007 (2015).

[24] S. P. Adhya, M. Mandal, S. Biswas, and P. K. Roy, Phys. Rev. D 93, 074033 (2016).

[25] G. Colucci, E. S. Fraga, and A. Sedrakian, Phys. Lett. B 728, 19 (2014).

[26] S. P. Adhya, M. Mandal, S. Biswas, and P. K. Roy, Phys. Rev. D 93, 074033 (2016).

[27] I. S. Gradshteyn and I. M. Rizhik, Table of Integrals 7th ed. (Elsevier, New York, 2007).

[28] E. J. Ferrer, V. de la Incera, D. M. Paret, A. P. Martinez, and A. Sanchez, Phys. Rev. D 91, 085041 (2015).

[29] R. J. Perry, Phys. Lett. B 199, 489 (1987).

[30] T. D. Cohen, M. K. Banerjee, and C.-Y. Ren, Phys. Rev. C 36, 1653 (1987).

[31] J. Caro, E. Ruiz Arriola, and L. L. Salcedo, Phys. Rev. C 55, 1767 (1997). 